\title{
Inclusive measurements of inelastic electron and positron scattering from unpolarized hydrogen and deuterium targets
}

\section{The HERMES Collaboration \\ E-mail: klaus.rith@desy.de}

ABSTRACT: Results of inclusive measurements of inelastic electron and positron scattering from unpolarized protons and deuterons at the HERMES experiment are presented. The structure functions $F_{2}^{p}$ and $F_{2}^{d}$ are determined using a parameterization of existing data for the longitudinal-to-transverse virtual-photon absorption cross-section ratio. The HERMES results provide data in the ranges $0.006 \leq x \leq 0.9$ and $0.1 \mathrm{GeV}^{2} \leq Q^{2} \leq 20 \mathrm{GeV}^{2}$, covering the transition region between the perturbative and the non-perturbative regimes of QCD in a so-far largely unexplored kinematic region. They are in agreement with existing world data in the region of overlap. The measured cross sections are used, in combination with data from other experiments, to perform fits to the photon-nucleon cross section using the functional form of the ALLM model. The deuteron-to-proton cross-section ratio is also determined.

KEYwORDS: Lepton-Nucleon Scattering

ARXIV EPRINT: 1103.5704 


\section{Contents}

1 Introduction 1

2 Formalism 2

3 The experiment $\quad 4$

4 Data analysis $\quad 5$

4.1 Event selection 5

$\begin{array}{lll}4.2 & \text { Trigger } & 7\end{array}$

$\begin{array}{lll}4.3 & \text { Particle identification } & 7\end{array}$

4.4 Charge-symmetric background 8

4.5 Luminosity 9

$\begin{array}{lll}4.6 & \text { Instrumental smearing and radiative effects } & 10\end{array}$

$\begin{array}{lll}4.7 & \text { Detection efficiency of specific radiative events } & 11\end{array}$

$\begin{array}{ll}4.8 \text { Misalignment effects } & 11\end{array}$

5 Systematic uncertainties $\quad 12$

$\begin{array}{lll}5.1 & \text { Inclusive inelastic scattering cross sections } & 12\end{array}$

$\begin{array}{ll}5.2 & \text { Inclusive inelastic scattering cross-section ratio } \sigma^{d} / \sigma^{p} \\ \end{array}$

6 Discussion of the results 13

$\begin{array}{lll}\text { 6.1 Structure functions } F_{2}^{p} \text { and } F_{2}^{d} & 13\end{array}$

$\begin{array}{lll}6.2 & \text { Cross-section ratio } \sigma^{d} / \sigma^{p} & 18\end{array}$

$\begin{array}{lll}6.3 & \text { Fits to world data to the cross section } \sigma_{L+T}^{p, d} & 18\end{array}$

6.4 Fits to world data for the cross-section ratio $\sigma^{d} / \sigma^{p} \quad 24$

$\begin{array}{lll}7 & \text { Summary } & 25\end{array}$

$\begin{array}{lr}\text { A Tables } & 26\end{array}$

\section{Introduction}

Over the past decades, lepton-nucleon scattering has played a major role in the development of our present understanding of nucleon structure. For a review on the subject see for example [1]. In lowest order perturbation theory, scattering of charged leptons $l$ off nucleons $N$ proceeds via the exchange of a neutral boson $\left(\gamma^{*}, Z^{0}\right)$. At the HERMES lepton-nucleon centre-of-mass energy of $\sqrt{s}=7.2 \mathrm{GeV}$, contributions from $Z^{0}$-exchange to the cross section can be neglected. Therefore, only the electromagnetic interaction in the approximation of 
one-photon exchange is considered here. In this approximation, the differential cross section of unpolarized inclusive charged-lepton-nucleon scattering, $l+N \rightarrow l^{\prime}+X$ (where $X$ denotes the undetected final state), is parameterized by two structure functions $F_{1}\left(x, Q^{2}\right)$ and $F_{2}\left(x, Q^{2}\right)$. Here $x=Q^{2} / 2 M \nu$ is the Bjorken variable, with $-Q^{2}$ being the square of the four-momentum transferred by the virtual photon and $\nu$ its energy in the target rest frame. The variable $x$ is a measure for the inelasticity of the process with $0 \leq x \leq 1$, and $x=1$ for elastic scattering.

In the deep-inelastic scattering (DIS) regime, $\sqrt{Q^{2}}$ and $\nu$ are much larger than the typical hadronic scale, usually set to be the mass $M$ of the nucleon, and the invariant mass $W$ of the photon-nucleon system is much larger than the masses of nucleon resonances. In the Quark-Parton Model (QPM), the DIS process is viewed as the incoherent superposition of elastic lepton scattering from quasi-free point-like quarks of any flavor $q$. The variable $x$ can then be interpreted as the fraction of the longitudinal nucleon momentum carried by the struck quark in a frame where the nucleon moves with infinite momentum in the direction opposite to that of the virtual photon. In this picture, quark distribution functions $f_{q}\left(x, Q^{2}\right)$ describe the number density of quarks of flavor $q$ in a fast-moving nucleon at a given value of $\left(x, Q^{2}\right)$ and experimental values of $F_{2}\left(x, Q^{2}\right)$ have been used to constrain these. At low values of $Q^{2}$, where this picture of incoherent quasi-free scattering does not apply, phenomenological models have been developed (see e.g. refs. [2] and [3]) to describe the measured structure functions.

A wealth of unpolarized inclusive charged-lepton DIS data is available from the collider experiments H1 [4-8] and ZEUS [9-14] at HERA with lepton-nucleon centre-of-mass energies $\sqrt{s}$ up to $320 \mathrm{GeV}$, the muon experiments BCDMS [15], EMC [16], NMC [17] and E665 [18] $(\sqrt{s} \cong 12-31 \mathrm{GeV})$, experiments with electrons at SLAC [19] $(\sqrt{s} \leq 7 \mathrm{GeV})$ and at JLAB [20-23]) $(\sqrt{s} \leq 3.25 \mathrm{GeV})$. The HERMES experiment [24] at HERA collected a large data set for positron and electron scattering on a variety of nuclear targets, including the proton and deuteron data presented here. In particular, the HERMES data cover the transition region between the perturbative and non-perturbative regimes of QCD in a kinematic region so far largely unexplored by other experiments. In this work, these data are presented together with fits to the world data for the photon-nucleon cross section using the Regge-motivated approach of the ALLM [3, 25] model. The paper is organized as follows: the formalism leading to the extraction of the structure function $F_{2}$ is briefly reviewed in section 2; section 3 deals with the HERMES experimental arrangement and the data analysis is described in section 4 . The systematic uncertainties in the resulting cross sections and cross-section ratios are discussed in section 5. Section 6 offers a discussion of the results and section 7 provides a summary.

\section{Formalism}

In the approximation of one-photon exchange, the inclusive differential cross section for scattering unpolarized charged leptons on unpolarized nucleons can be conveniently pa- 


\begin{tabular}{|l|l|}
\hline$m_{l}$ & $\begin{array}{l}\text { Lepton mass (taken to be negligible) } \\
\text { Mass of target nucleon }\end{array}$ \\
$k=(E, \vec{k}), k^{\prime}=\left(E^{\prime}, \overrightarrow{k^{\prime}}\right)$ & 4-momenta of the initial and final state leptons \\
$\theta, \phi$ & Polar and azimuthal angle of the scattered lepton \\
$P \stackrel{\text { lab }}{=}(M, 0)$ & $\begin{array}{l}\text { 4-momentum of the initial target nucleon } \\
\text { 4-momentum of the virtual photon }\end{array}$ \\
$Q^{2}=-q^{2} \stackrel{\text { lab }}{\approx} 4 E E^{\prime} \sin ^{2} \frac{\theta}{2}$ & $\begin{array}{l}\text { Negative squared 4-momentum transfer } \\
\text { Energy of the virtual photon in the target rest } \\
\text { frame } \\
M=\frac{P \cdot q}{M} \stackrel{\text { lab }}{=} E-E^{\prime} \\
x=\frac{Q^{2}}{2 P \cdot q}=\frac{Q^{2}}{2 M \nu}\end{array}$ \\
$y=\frac{P \cdot q}{P \cdot k} \stackrel{\text { lab }}{=} \frac{\nu}{E}$ & $\begin{array}{l}\text { Bjorken scaling variable } \\
\text { Fractional energy of the virtual photon } \\
W^{2}=(P+q)^{2}=M^{2}+2 M \nu-Q^{2}\end{array}$ \\
\hline
\end{tabular}

Table 1. Kinematic variables used in the description of lepton scattering.

rameterized in terms of the structure functions $F_{1}\left(x, Q^{2}\right)$ and $F_{2}\left(x, Q^{2}\right)$ :

$$
\frac{d^{2} \sigma}{d x d Q^{2}}=\frac{4 \pi \alpha_{\mathrm{em}}^{2}}{Q^{4}}\left[y^{2} \cdot F_{1}\left(x, Q^{2}\right)+\left(\frac{1-y}{x}-\frac{M y}{2 E}\right) \cdot F_{2}\left(x, Q^{2}\right)\right],
$$

where $\alpha_{\mathrm{em}}$ is the fine-structure constant and all other variables are described in table 1 . The quantities $x$ and $Q^{2}$ are fully determined by the kinematic conditions of the incident and scattered leptons and the target nucleon. This cross section can also be written in terms of longitudinal $(L)$ and transverse $(T)$ virtual-photon contributions

$$
\frac{d^{2} \sigma}{d x d Q^{2}}=\Gamma\left[\sigma_{T}\left(x, Q^{2}\right)+\epsilon \sigma_{L}\left(x, Q^{2}\right)\right]
$$

where $\sigma_{L}$ and $\sigma_{T}$ are the absorption cross sections for longitudinal and transverse virtual photons, $\Gamma$ is the flux of transverse virtual photons and the virtual-photon polarization parameter $\epsilon$ is the ratio of virtual-photon fluxes for longitudinal and transverse polarizations [26]. The structure functions $F_{1}\left(x, Q^{2}\right)$ and $F_{2}\left(x, Q^{2}\right)$ can then be expressed in terms 
of the two virtual-photon absorption cross sections $\sigma_{L}\left(x, Q^{2}\right)$ and $\sigma_{T}\left(x, Q^{2}\right)$ :

$$
\begin{aligned}
& F_{1}\left(x, Q^{2}\right)=\frac{1}{4 \pi^{2} \alpha_{\mathrm{em}}} M K \cdot \sigma_{T}\left(x, Q^{2}\right), \\
& F_{2}\left(x, Q^{2}\right)=\frac{1}{4 \pi^{2} \alpha_{\mathrm{em}}} \frac{\nu K}{1+\frac{Q^{2}}{4 M^{2} x^{2}}} \cdot\left[\sigma_{L}\left(x, Q^{2}\right)+\sigma_{T}\left(x, Q^{2}\right)\right],
\end{aligned}
$$

where $K=\nu(1-x)$ in the Hand convention [27, 28]. The longitudinal-to-transverse photon-absorption cross-section ratio $R=\sigma_{L} / \sigma_{T}$ can be expressed in terms of $F_{1}$ and $F_{2}$ :

$$
R\left(x, Q^{2}\right)=\frac{\sigma_{L}}{\sigma_{T}}=\left(1+\frac{4 M^{2} x^{2}}{Q^{2}}\right) \frac{F_{2}\left(x, Q^{2}\right)}{2 x F_{1}\left(x, Q^{2}\right)}-1 .
$$

A determination of the structure functions $F_{1}\left(x, Q^{2}\right)$ and $F_{2}\left(x, Q^{2}\right)$ requires in principle cross-section measurements made at the same $x$ and $Q^{2}$ but at two or more different values of $y$ (see eq. (2.1)), i.e., with different beam energies. The HERMES data used for this analysis were taken at a single beam energy. In such a situation, it is common practice to re-parameterize the cross section, eq. (2.1), as a function of $F_{2}$ and $R$ using eq. (2.5):

$$
\frac{d^{2} \sigma}{d x d Q^{2}}=\frac{4 \pi \alpha_{\mathrm{em}}^{2}}{Q^{4}} \frac{F_{2}\left(x, Q^{2}\right)}{x}\left[1-y-\frac{Q^{2}}{4 E^{2}}+\frac{y^{2}+Q^{2} / E^{2}}{2\left[1+R\left(x, Q^{2}\right)\right]}\right] .
$$

The structure function $F_{2}\left(x, Q^{2}\right)$ can then be extracted from a single cross-section measurement at a given $\left(x, Q^{2}\right)$, by using a parameterization for $R\left(x, Q^{2}\right)$ obtained from the available world data. This approach has been used in the analysis presented in this paper.

\section{The experiment}

The HERA facility at DESY comprised a proton and a lepton storage ring. HERMES was a fixed-target experiment using only the lepton beam, which consisted of either electrons or positrons at an energy of $27.6 \mathrm{GeV}$, while the proton beam passed through the noninstrumented horizontal mid-plane of the HERMES spectrometer. An open-ended storage cell that could be fed with either polarized or unpolarized gas was installed internally to the lepton ring.

The HERMES spectrometer, which consisted of two identical halves above and below the electron beam, was a forward spectrometer [24] with multiple tracking stages before and after a $1.5 \mathrm{Tm}$ dipole magnet. It had a geometrical acceptance of $\pm 170 \mathrm{mrad}$ horizontally and $\pm(40-140)$ mrad vertically for particles originating from the center of the target cell, resulting in polar scattering angles $\theta$ ranging from about 40 to 220 mrad. Particle identification (PID) capabilities were provided by combining the responses of a lead-glass calorimeter, a pre-shower hodoscope (H2), a transition-radiation detector (TRD), and a threshold Čerenkov detector that was upgraded to a dual-radiator ring-imaging Cerenkov detector (RICH) $[29,30]$ in the year 1998. The lead-glass calorimeter and the pre-shower hodoscope were included in the trigger together with two other hodoscopes (H0 and H1). 
In this experiment target gases of hydrogen and deuterium were used. Part of the data were taken with polarized hydrogen and deuterium, with the target spin being reversed in 13 min time intervals so that the target was effectively unpolarized. In the case of hydrogen, using areal densities of the order of $10^{14}$ nucleons $\mathrm{cm}^{-2}$ and lepton currents of typically about $30 \mathrm{~mA}$, luminosities of the order of $2 \cdot 10^{31} \mathrm{~cm}^{-2} \mathrm{~s}^{-1}$ were achieved for the polarized running, and about 10 times higher values for unpolarized running. The luminosity was measured by scattering the lepton beam off the atomic electrons of the target gas, i.e., Møller scattering $e^{-} e^{-} \rightarrow e^{-} e^{-}$for an electron beam and Bhabha scattering $e^{+} e^{-} \rightarrow e^{+} e^{-}$ together with the annihilation process $e^{+} e^{-} \rightarrow \gamma \gamma$ for a positron beam. The cross sections for these processes are precisely known in Quantum Electrodynamics, including radiative corrections. The scattered particles were detected in coincidence by two identical small calorimeters [31] located symmetrically with respect to the beam pipe. The coincidence rate of the pairs of leptons (and photons) provided a relative monitor of the luminosity. An absolute calibration of the luminosity measurement was provided by correlating the coincidence rate with the yields of the Møller, Bhabha and annihilation processes.

\section{Data analysis}

An event is accepted if it contains a track identified as a lepton by the PID system (see section 4.3), and satisfies the selection criteria described in section 4.1. The number of measured events $N_{\text {meas }}$ in each $\left(x, Q^{2}\right)$ bin is corrected by subtracting the charge-symmetric background from secondary processes $N_{\mathrm{cs}}$ and by dividing the resulting number by the corresponding trigger and lepton-identification efficiencies $\mathcal{E}_{\text {trigger }}$ and $\mathcal{E}_{\text {lep }}$, while taking into account the hadron contamination $\mathcal{C}_{\text {had }}$ :

$$
N_{\text {events }}=\left(N_{\text {meas }}-N_{\mathrm{cs}}\right) \cdot \frac{1}{\mathcal{E}_{\text {trigger }}} \cdot \frac{1-\mathcal{C}_{\text {had }}}{\mathcal{E}_{\text {lep }}} .
$$

These corrections are described in sections 4.2 to 4.4 .

The experimental cross section is then obtained as the ratio of the number of events $N_{\text {events }}$ in each $\left(x, Q^{2}\right)$ bin of widths $\Delta x$ and $\Delta Q^{2}$, and the integrated luminosity $L$ (see section 4.5):

$$
\frac{d^{2} \sigma_{\operatorname{Exp}}}{d x d Q^{2}}\left(x, Q^{2}\right)=\frac{N_{\text {events }}\left(x, Q^{2}\right)}{\Delta x \Delta Q^{2}} \cdot \frac{1}{L} .
$$

An unfolding procedure for disentangling instrumental and radiative effects from the measured cross section is then applied in order to obtain the Born cross sections $\sigma_{\text {Born }}^{p, d} \equiv \sigma^{p, d}$ (see section 4.6). The structure functions $F_{2}^{p}$ and $F_{2}^{d}$ are finally derived from the Born cross sections through eq. (2.6) using the parameterization $R=R_{1998}$ [32]. Two more corrections related to detector geometry and alignment are discussed in sections 4.7 and 4.8.

\subsection{Event selection}

The kinematic range of the events selected for this analysis is shown in figure 1, together with the requirements imposed on the kinematic and geometrical variables. The tracks are required to be fully contained within the fiducial geometric acceptance of the 


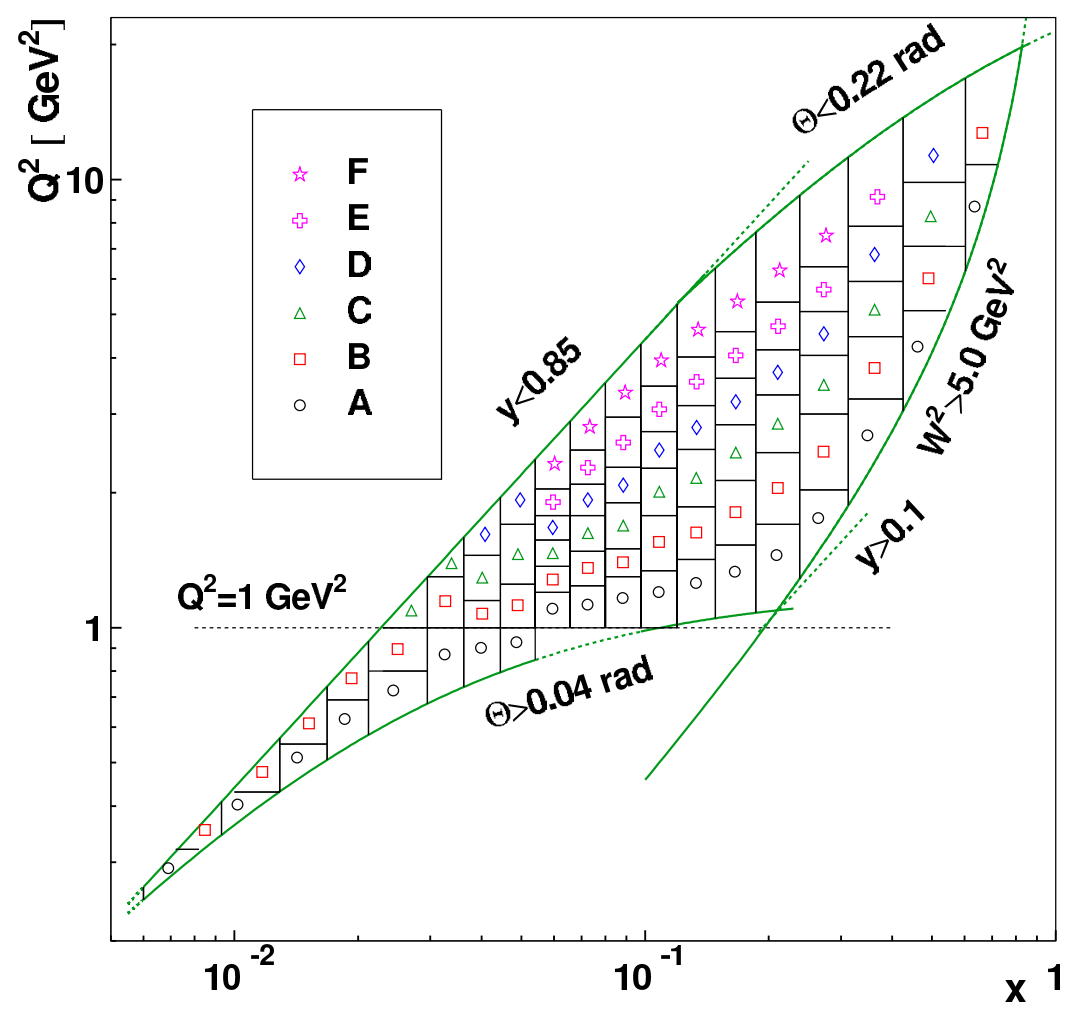

Figure 1. Binning in $\left(x, Q^{2}\right)$ used in the analysis and kinematic acceptance of events. The kinematic region covered is limited by the geometrical acceptance in $\theta$ and constraints on $y$ and $W^{2}$. The symbols mark the locations of the average values of $\left(x, Q^{2}\right)$ of each bin. The symbols $\mathrm{A}$ to $\mathrm{F}$ denote bins with increasing $Q^{2}$ at given $x$.

\begin{tabular}{|r|r|r|}
\hline Year & \multicolumn{2}{|c|}{ events, in million } \\
\hline & proton & deuteron \\
\hline 1996 & 2.3 & 2.8 \\
1997 & 4.6 & 3.1 \\
2000 & 9.5 & 12.6 \\
\hline total & 16.4 & 18.5 \\
\hline
\end{tabular}

Table 2. Number of raw events $N_{\text {meas }}$ used in this analysis, separated into the years of data taking. The numbers correspond to the total luminosities of about $450 \mathrm{pb}^{-1}$ on the proton and about $460 \mathrm{pb}^{-1}$ on the deuteron.

HERMES spectrometer. The constraint $W^{2}>5 \mathrm{GeV}^{2}$ excludes the region of nucleon resonances and acts as a selection for $y>0.1$. The constraint $y \leq 0.85$ discards the low-momentum region, where radiative effects increase and where the trigger efficiency has not yet reached its plateau. The requirements imposed on $y$ select the momentum range $4.1 \mathrm{GeV}<p<24.8 \mathrm{GeV}$ for the detected particle. The resulting $\left(x, Q^{2}\right)$ region, $0.006 \leq x \leq 0.9$ and $0.1 \mathrm{GeV}^{2} \leq Q^{2} \leq 20 \mathrm{GeV}^{2}$, is subdivided into 19 bins in $x$ and each $x$ bin into up to six bins in $Q^{2}$. 


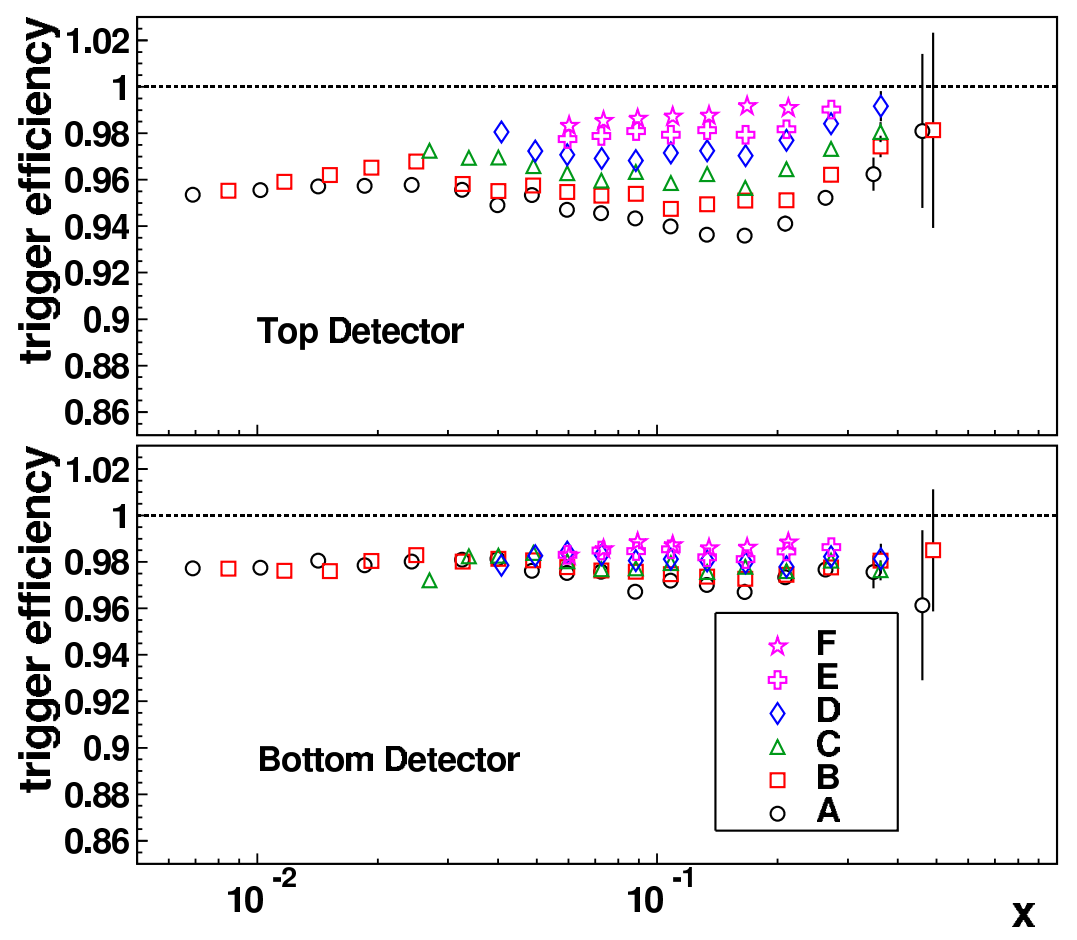

Figure 2. Trigger efficiencies $\mathcal{E}_{\text {trigger }}$ for data taken in the year 2000 shown separately for the top and bottom spectrometer halves. The error bars represent only statistical uncertainties. The symbols refer to the $Q^{2}$ bins shown in figure 1 .

Table 2 shows the numbers of events for each year of measurements used in this analysis, before the application of any of the corrections discussed in the following sections.

\subsection{Trigger}

The trigger used for the recording of inelastic scattering events required signals from the hodoscopes H0, H1, and $\mathrm{H} 2$ and a sufficiently large energy deposition in the calorimeter. The efficiencies of the trigger detectors are extracted individually from special calibration triggers and combined to obtain the total trigger efficiency. It is assumed that inefficiencies in the electronic trigger logics are negligible. Trigger efficiencies are sensitive to, among others, misalignment effects, detector-voltage setting, and radiation damage, the latter especially in the H0 hodoscope. This last effect is responsible for the reduced efficiencies seen at small scattering angles and for the differences between the top and bottom detector. Such differences are shown in figure 2 for data taken in the year 2000, for the kinematic binning used in the analysis.

\subsection{Particle identification}

The scattered lepton (positron or electron) is identified by a combination of the responses of the transition-radiation detector TRD, the pre-shower hodoscope $\mathrm{H} 2$, and the leadglass calorimeter. Each of these elements used alone gives a high rejection of hadrons. A Čerenkov detector provides additional hadron identification. (A threshold Čerenkov was 


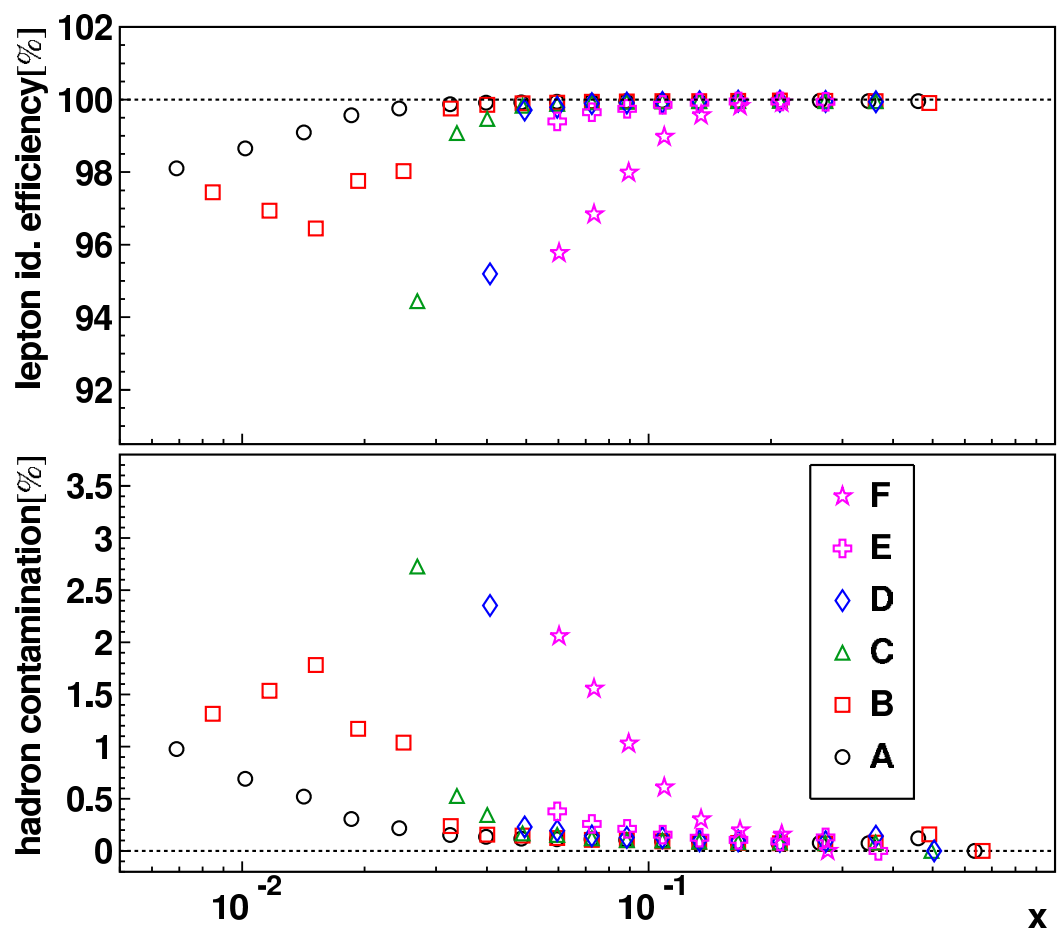

Figure 3. Lepton identification efficiency $\mathcal{E}_{\text {lep }}$ and hadron contamination $\mathcal{C}_{\text {had }}$ in the year 2000. The symbols refer to the $Q^{2}$ binning shown in figure 1.

used for pion identification in 1996-97, and a ring-imaging Čerenkov detector was used thereafter to identify pions, kaons, and protons.) The detector response of an individual PID element is determined by placing very restrictive constraints on the response of the remaining elements, thereby generating a clean sample of a given kind of particles with which the unit under study is calibrated.

In combination, the array of detectors provides an average lepton identification with an efficiency $\mathcal{E}_{\text {lep }}$ of about $98 \%$ and an average hadron contamination $\mathcal{C}_{\text {had }}$ below $1 \%$ over the full kinematic range of the HERMES acceptance. At low values of $x$, lepton efficiencies as low as $94 \%$ and hadron contaminations as high as $3 \%$ are reached.

The efficiency for lepton identification and the fractional hadron contamination as a function of $x$ for the various $Q^{2}$ bins are presented in figure 3 for representative data taken in the year 2000. The figure shows that for smaller values of $x(x<0.1)$ a lower lepton identification efficiency appears correlated to a larger hadron contamination.

\subsection{Charge-symmetric background}

The observed event sample is contaminated by background coming mostly from chargesymmetric processes, such as meson Dalitz decays (e. g. $\pi^{0} \rightarrow e^{+} e^{-} \gamma$ ) or photon conversions into $e^{+} e^{-}$pairs. Since these positrons and electrons originate from secondary processes, they typically have lower momenta and are thus concentrated at high $y$. A correction for charge-symmetric background events $N_{\mathrm{cs}}$ is applied in each kinematic bin by counting with negative weight leptons with a charge opposite to that of the beam particle. It is assumed 


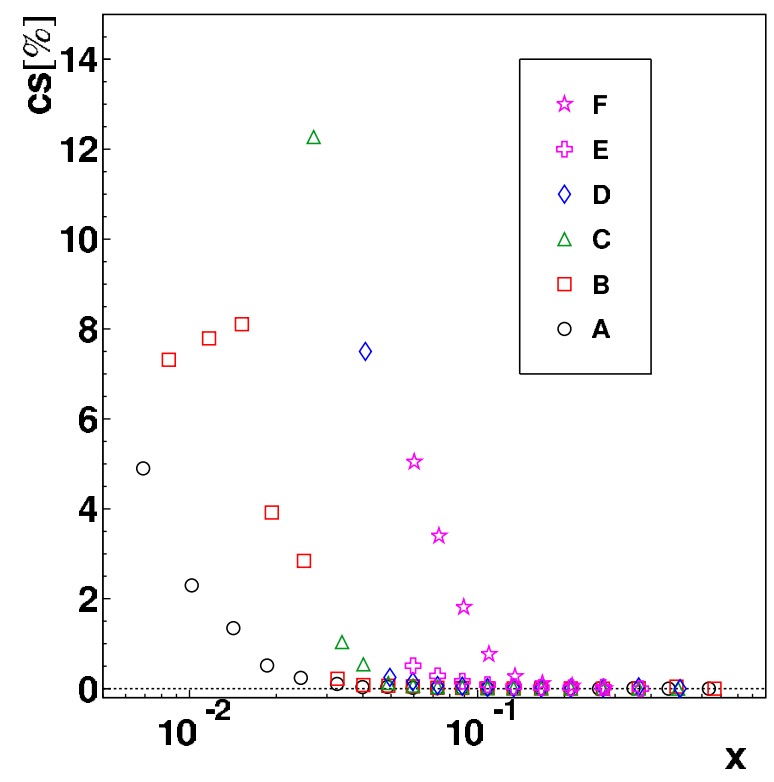

Figure 4. Percentage of charge-symmetric background, calculated from the ratio of the chargesymmetric events to the total events in each bin, for the 2000 deuterium data. The symbols refer to the $Q^{2}$ binning shown in figure 1 .

that acceptance and inefficiencies are the same for background electrons and positrons, even though their spatial distributions after the magnet are quite different. The $x$ dependence of CS, the ratio of charge-symmetric events to the total number of events in each kinematic bin, is shown for the six $Q^{2}$ bins in figure 4 . The charge-symmetric background is negligible at large particle momenta, but reaches up to $12 \%$ at low particle momenta of about $6 \mathrm{GeV}$.

\subsection{Luminosity}

The integrated luminosity $L$ per nucleon is calculated as flollows:

$$
L=\int \mathcal{L} d t=\left(R_{\mathrm{LR}}-2 \Delta t \cdot R_{L} \cdot R_{R}\right) \cdot c_{\text {live }} \cdot C_{\mathrm{Lumi}} \cdot \Delta b \cdot \frac{A}{Z} .
$$

Here, $R_{L}$ and $R_{R}$ are the count rates in the left and right luminosity detector, respectively, $R_{\mathrm{LR}}$ is the coincidence rate measured within a time window of $\Delta t=40 \mathrm{~ns}, c_{\text {live }}$ is the trigger livetime factor, $C_{\mathrm{Lumi}}$ is the year-dependent luminosity factor, $\Delta b$ is the time interval in which the luminosity rates were obtained, and $A / Z$ is the ratio of the numbers of nucleons $(A)$ and electrons $(Z)$ in the target gas atoms. The term $2 \Delta t \cdot R_{L} \cdot R_{R}$ in eq. (4.3) corrects for accidental coincidences according to the statistical expectation and is of the order of $0.1-0.5 \%$. The physics trigger livetime contribution $c_{\text {live }}$ is defined as the fraction of the physics events that are accepted by the data acquisition system out of all events generating a physics trigger. This quantity is typically above $90 \%$.

The data aquisition system of the luminosity detector worked independently of the physics triggers. It is assumed that the inefficiency of the luminosity event trigger was negligible. The luminosity factor $C_{\text {Lumi }}$ accounts for the geometric acceptance of the luminosity detector, the beam position and the absolute Møller and Bhabha cross sections. 
Its year dependence derives from the ageing of the luminosity detector and different running conditions, i.e., changes in beam charge and beam optics. The dependence of the coincidence rate from beam position and slope was measured in order to disentangle the dependence of the measured coincidence rate from beam orbits and geometrical acceptance of the luminosity detector. The uncertainty in the measurement of the absolute luminosity is dominated by the uncertainty on the acceptance of the detector, which depends sensitively on the impact coordinates of the particle. The uncertainty on the latter is about $2.5 \mathrm{~mm}$, which propagates into an uncertainty of about $7 \%$ on the integrated cross section and therefore on the luminosity.

\subsection{Instrumental smearing and radiative effects}

Instrumental smearing is due to intrinsic detector resolution and multiple scattering in the various detector elements of the particles emerging from the DIS process and identified as the scattered lepton. Radiative effects include vertex corrections to the QED hard scattering amplitude and radiation of one or more real photons by the incoming or outgoing lepton. Radiative effects and instrumental smearing both modify the Born kinematic conditions resulting in altered reconstructed kinematic variables. Migration probabilities for the relevant kinematic variables are determined from a Monte Carlo simulation and used to correct the measured distributions.

The Born cross section for inelastic scattering on the proton is simulated according to the ALLM97 parameterization of $F_{2}^{p}[25]$ and the parameterization $R_{1990}$ [33] for $R$, while that for the deuteron is derived from the same parameterizations in conjunction with the fit [34] to $F_{2}^{d} / F_{2}^{p}$ data from NMC, SLAC and BCDMS. Radiative effects are simulated with RADGEN [35]. The electric and magnetic form factors of the proton and neutron, from which the elastic cross sections are derived, are taken from the fits in refs. [36] and [37]. When using the more recent parameterizations for the proton from ref. [38], the results are essentially the same.

The probabilistic information about event migration can be summarized in a smearing matrix [39],

$$
S(i, j)=\frac{\partial \sigma_{\operatorname{Exp}}(i)}{\partial \sigma_{\operatorname{Born}}(j)}=\frac{n(i, j)}{n_{\operatorname{Born}}(j)}
$$

Here, $n(i, j)$ is the migration matrix representing the number of events originating from kinematic bin $j$ at Born level and measured in bin $i$. It is extracted from a Monte Carlo simulation with full track reconstruction that simulates the inelastic scattering cross section, QED radiative effects and instrumental smearing. Material outside the detector acceptance is excluded from this simulation for computational economy. The vector $n_{\text {Born }}(j)$ containing the number of events at Born level is obtained from a second Monte Carlo calculation that simulates only the (unradiated) inelastic cross section. An additional column $j=0$ is defined for events that migrate into the acceptance from outside. The smearing matrix $S(i, j)$ has the property of being independent from the generated cross section within the acceptance. 
The inverted squared submatrix $S^{\prime}(i, j)=S(i, j>0)$ relates the measured distributions to the distributions at Born level:

$$
\sigma_{\mathrm{Born}}(j)=\sum_{i} S^{-1}(j, i) \times\left[\sigma_{\operatorname{Exp}}(i)-S(i, 0) \sigma_{\mathrm{Born}}(0)\right] .
$$

The reconstruction of simulated tracks uses the same algorithm as for real data. Tracking-related inefficiencies are taken into account in the unfolding procedure, assuming that coincident particles outside the acceptance do not significantly affect the efficiency and the simulation adequately models the physical processes in the tracking detectors.

The Monte Carlo generated data sample was a factor 10 larger than the experimental data sample. The statistical uncertainties of the Monte Carlo data enter mainly via the simulated experimental count rates in the migration matrices. A multi-sampling numerical approach is used to propagate these statistical uncertainties through the unfolding algorithm. The statistical uncertainties of the inelastic scattering Born cross section coming from the experimental cross section and those originating from the finite statistical precision of the Monte Carlo are summed in quadrature to produce the total statistical uncertainty.

\subsection{Detection efficiency of specific radiative events}

Radiative corrections include cases where the incoming electron radiates a high-energy photon and then scatters elastically from the nucleon with negligible momentum transfer. The efficiency to detect such events is reduced due to the following effect. The radiated photon is emitted at small angles and has a large probability to hit the beam pipe, generating an electromagnetic shower that saturates the wire chambers. This makes the data acquisition system skip the event as no tracking is possible. In order to compensate for this omission, the detection efficiency for elastic and quasi-elastic radiative events is estimated using a dedicated Monte Carlo simulation that includes a complete treatment of showers in material outside the geometrical acceptance.

The resulting efficiencies $\mathcal{E}_{\text {e.m. }}$ are significantly less than $100 \%$ in the range $0.01<x<$ 0.1 . They show a dip at $x \simeq 0.02$ where, in the case of the proton, they reach values as low as $80 \%$ while in the case of the deuteron they are as low as $90 \%(60 \%)$ for elastic (quasi-elastic) events. They are applied to the background term $S(i, 0) \sigma_{\mathrm{Born}}(0)$ in order to not over-correct for radiative processes that are not observed in the spectrometer. More details can be found in refs. [39, 40].

\subsection{Misalignment effects}

Imperfect alignment of the two spectrometer halves and the beam with respect to their ideal positions is studied in order to estimate the impact on the measured cross sections and structure functions. Misalignment effects cannot be corrected for in the unfolding because they are not of a stochastic nature. Rather, they are studied in a Monte Carlo simulation, and the fractional change of the Born cross section in each kinematic bin is obtained from the ratio of unfolded cross sections when using a MC with an aligned geometry and another with a misaligned geometry. These fractional changes are used to rescale the experimental Born cross sections on a bin-by-bin basis. Misalignment effects are most significant for 
small scattering angles and high particle momenta, i.e., at small $Q^{2}$ in each bin of $x$. The correction reaches values as high as $19 \%$ in the lowest $Q^{2}$ bin and decreases to about $3 \%$ in the highest $Q^{2}$ bin.

\section{$5 \quad$ Systematic uncertainties}

\subsection{Inclusive inelastic scattering cross sections}

Particle identification. Correlations between PID detectors as described in section 4.3 cannot be completely avoided. They may bias the correction for particle identification. These effects are covered by the assignment of a conservative PID uncertainty of the full size of the correction (see eq. (4.1)). Hadrons are predominantly produced at small momenta. Thus particle misidentification occurs more likely at high $y$, i.e., towards higher $Q^{2}$ in each bin of $x$. Nevertheless, the uncertainty due to particle identification, $\delta_{\text {PID }}$, is always smaller than $3 \%$, because contaminations somewhat compensate inefficiencies.

Instrumental smearing and radiative effects. In the unfolding procedure an uncertainty can arise from uncertainties in the formalism to calculate radiative effects and in the model used for the cross section outside the acceptance. The latter affect our results through the radiative tail. The uncertainty, $\delta_{\text {model }}$, was estimated by varying the input elastic and inelastic cross sections within their uncertainties and found to be below $2 \%$, except for a few bins, where it went up to $4.3 \%$ (3.1\%) at maximum for the proton (deuteron) case. This is negligible compared to the overall normalization uncertainty of our data of about $7 \%$ (see below).

Misalignment. In each bin, half of the deviations of yields obtained in a Monte Carlo simulation with estimated geometric misalignments from the yields obtained in a Monte Carlo simulation with aligned (ideal) geometry serve as an estimate of the systematic uncertainty due to misalignment. The uncertainty due to misalignment, $\delta_{\text {mis. }}$, reaches values of up to $5.4 \%$. However, the majority of the data points has an uncertainty due to misalignment that is smaller than $3 \%$.

Dependence on misalignment of the efficiency $\mathcal{E}_{\text {e.m. }}$ for elastic and quasi-elastic radiative events. The efficiency $\mathcal{E}_{\text {e.m. }}$ and its dependence on misalignment were studied in Monte Carlo simulations. The assignment of a corresponding systematic uncertainty is accomplished by applying the values of $\mathcal{E}_{\text {e.m. }}$ extracted from a Monte Carlo simulation with aligned and misaligned geometry to the high-multiplicity radiative events included in the background term $S(i, 0) \sigma_{\text {Born }}(0)$. The difference of the unfolded inelastic scattering Born cross sections obtained for these efficiencies is assigned as a systematic uncertainty, $\delta_{\text {rad. }}$, due to these radiative corrections.

Overall normalization uncertainty. The normalization uncertainties of the absolute cross sections and the structure functions are dominated by the uncertainty of the yeardependent luminosity constant $C_{\text {Lumi }}$ in eq. (4.3). The uncertainties of the luminosity 
constants weighted with the sizes of the data sets result in an overall normalization uncertainty of $7.6 \%$ for the data taken on a hydrogen target and $7.5 \%$ for the data taken on a deuterium target.

\subsection{Inclusive inelastic scattering cross-section ratio $\sigma^{d} / \sigma^{p}$}

The cross-section ratio can be determined with higher precision than the cross sections themselves due to the cancellation of the misalignment uncertainty, the PID uncertainty and, to a large extent, the overall normalization uncertainty. The remaining overall normalization uncertainy of $1.4 \%$ is attributed to variations of the beam conditions between data sets.

The efficiencies $\mathcal{E}_{\text {e.m. }}$ for proton and deuteron are different [41], and therefore do not cancel in the proton-to-deuteron cross-section ratio. The uncertainty $\delta_{\text {rad }}$ of the crosssection ratio is obtained by propagating, for proton and deuteron cross sections, the uncertainties of efficiencies for high-multiplicity radiative events due to misalignment. It is found to be less than $2.5 \%$ in every kinematic bin.

\section{Discussion of the results}

The kinematic conditions of the HERMES inclusive lepton-nucleon scattering cross sections presented here overlap those of existing data over a large kinematic range. New information is provided in the region with $Q^{2} \lesssim 1 \mathrm{GeV}^{2}$ and $15 \mathrm{GeV}^{2}<W^{2}<45 \mathrm{GeV}^{2}$, corresponding to $0.006<x<0.04$.

\subsection{Structure functions $F_{2}^{p}$ and $F_{2}^{d}$}

The differential cross sections $d^{2} \sigma^{p, d} / d x d Q^{2}$ for inelastic scattering on the proton and deuteron as well as the corresponding structure functions $F_{2}^{p}$ and $F_{2}^{d}$ are listed in tables 5 and 6 together with the statistical and systematic uncertainties. The statistical uncertainties of the HERMES data range between $0.4 \%$ and $3.0 \%$. Almost $80 \%$ of all data points have a statistical uncertainty smaller than $1 \%$. The overall normalization uncertainty of $7.6 \%(7.5 \%)$ for the inelastic scattering cross section on the proton (deuteron) and the contribution from misalignment are the dominating systematic uncertainties.

The differential cross sections are shown in figures 5 and 6 as a function of $Q^{2}$ in bins of $x$. The structure functions are shown in figures 7 and 8 , together with the available world data from fixed target (E665 [18], BCDMS [15], NMC [17], SLAC [19], JLAB [2023]) and collider experiments (H1 and ZEUS) [44]. The data are overlaid with new fits to world data, including the data presented here, of inclusive proton (GD11-P) and deuteron (GD11-D) cross sections. These functions are described in section 6.3.

In the region $x \geq 0.07$ and $Q^{2}>1 \mathrm{GeV}^{2}$, HERMES data are in good agreement with existing data from SLAC and NMC. The HERMES measurement provides also data in a previously uncovered kinematic region between JLAB data on the one hand and NMC, BCDMS, E665 and the collider experiments on the other. This can be clearly seen in figures 7 and 8 . 


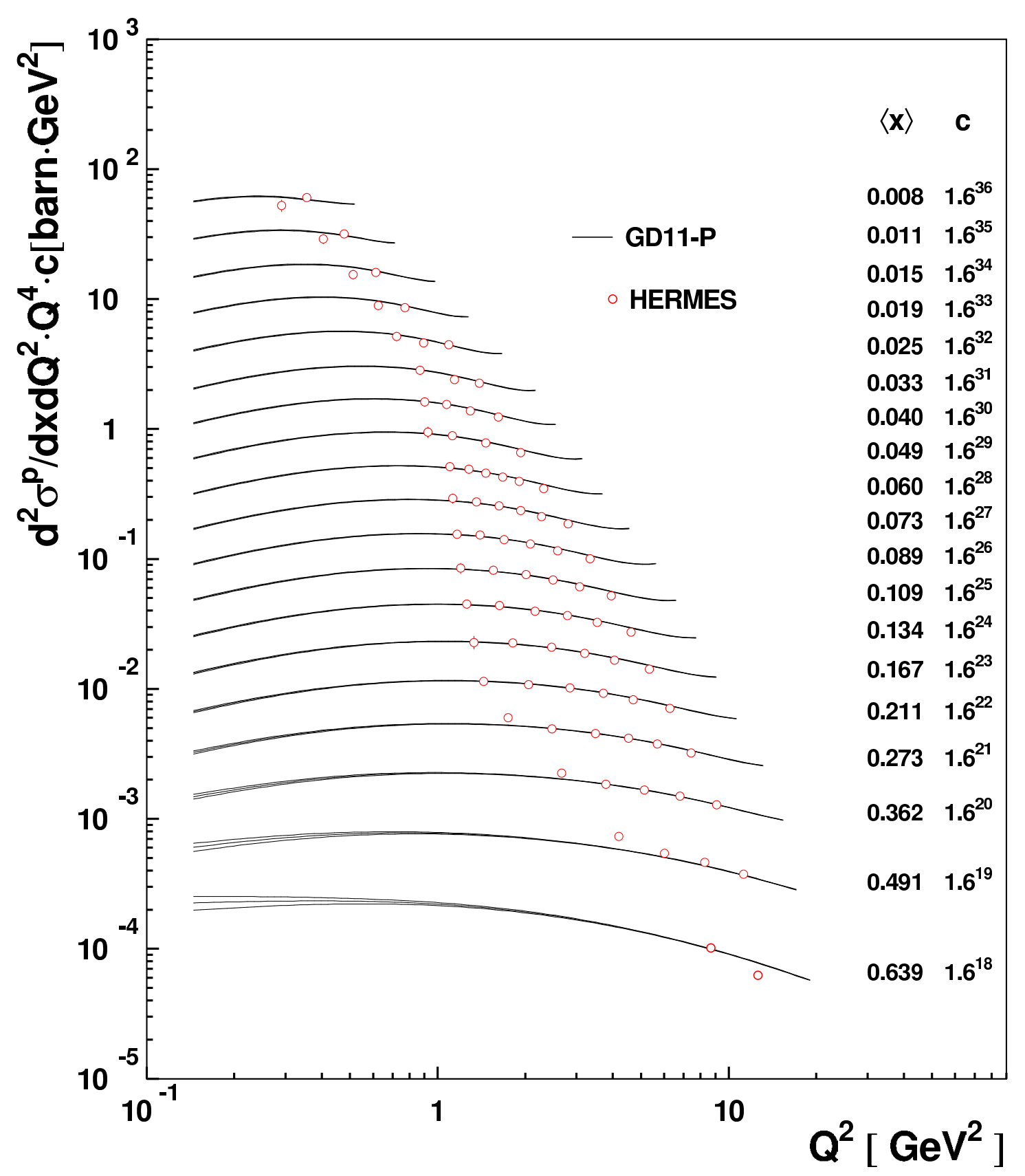

Figure 5. Inelastic proton differential DIS cross section $d^{2} \sigma^{p} / d x d Q^{2}$, multiplied by a factor $Q^{4}$ for the purpose of illustration, in the kinematic range $0.008 \leq\langle x\rangle \leq 0.639$ and $0.2 \mathrm{GeV}^{2} \leq\left\langle Q^{2}\right\rangle \leq$ $20 \mathrm{GeV}^{2}$. The values of $d^{2} \sigma^{p} / d x d Q^{2} \cdot Q^{4}$ are scaled by powers of 1.6 . The results are overlaid with the phenomenological parameterization GD11-P (central curves) and its uncertainty (outer curves). Details on the fits can be found in section 6.3. The error bars represent the total uncertainties calculated as the sum in quadrature of all statistical and systematic uncertainties including normalization, and are smaller than the symbols. 


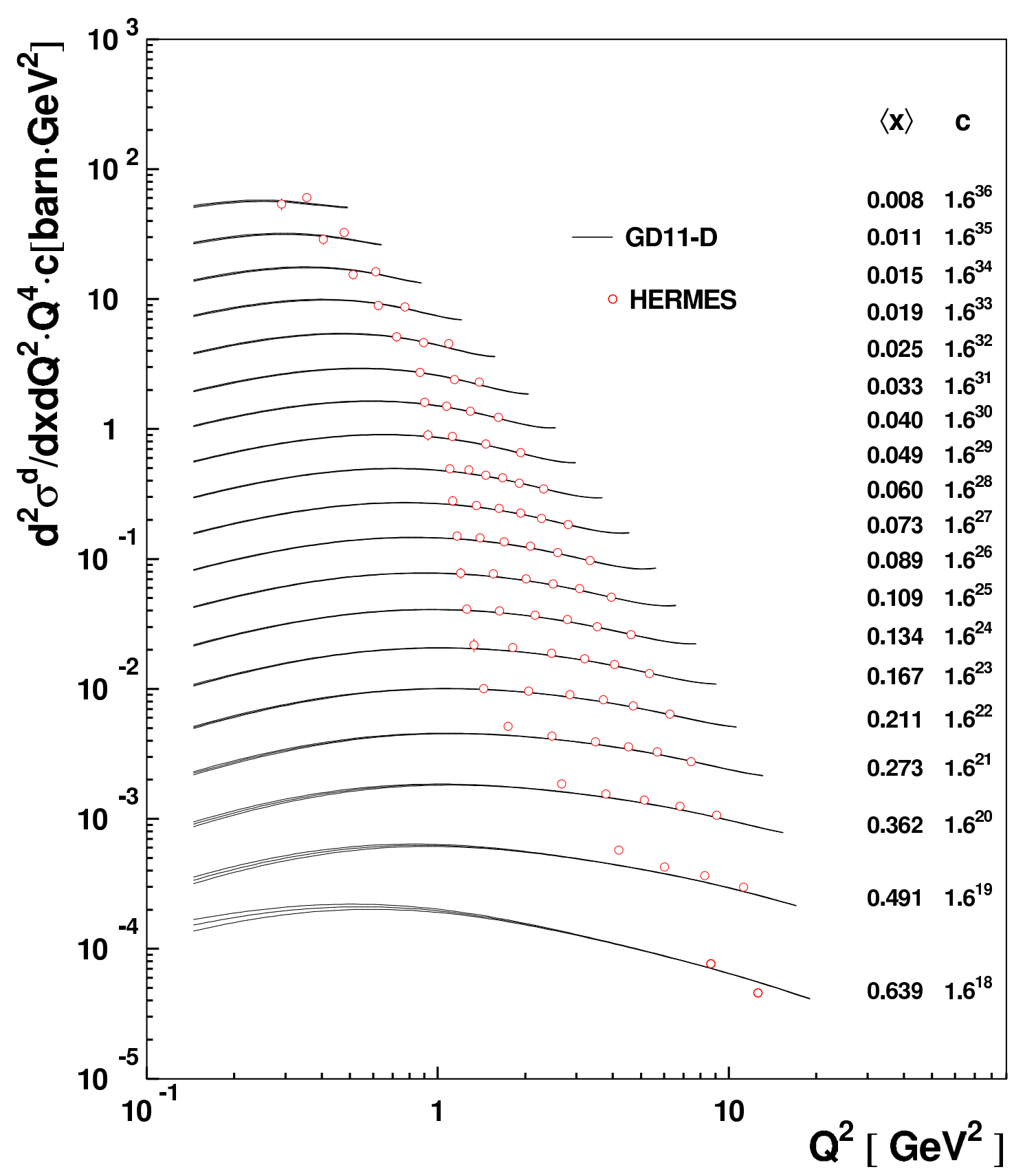

Figure 6. Inelastic deuteron differential DIS cross section $d^{2} \sigma^{d} / d x d Q^{2}$, multiplied by a factor $Q^{4}$ for the purpose of illustration, in the kinematic range $0.008 \leq\langle x\rangle \leq 0.639$ and $0.2 \mathrm{GeV}^{2} \leq$ $\left\langle Q^{2}\right\rangle \leq 20 \mathrm{GeV}^{2}$. The values of $d^{2} \sigma^{d} / d x d Q^{2} \cdot Q^{4}$ are scaled by powers of 1.6 . The results are overlaid with the phenomenological parameterization GD11-D (central curves) and its uncertainty (outer curves). Details on the fits can be found in section 6.3. The error bars represent the total uncertainties calculated as the sum in quadrature of all statistical and systematic uncertainties including normalization, and are smaller than the symbols. 


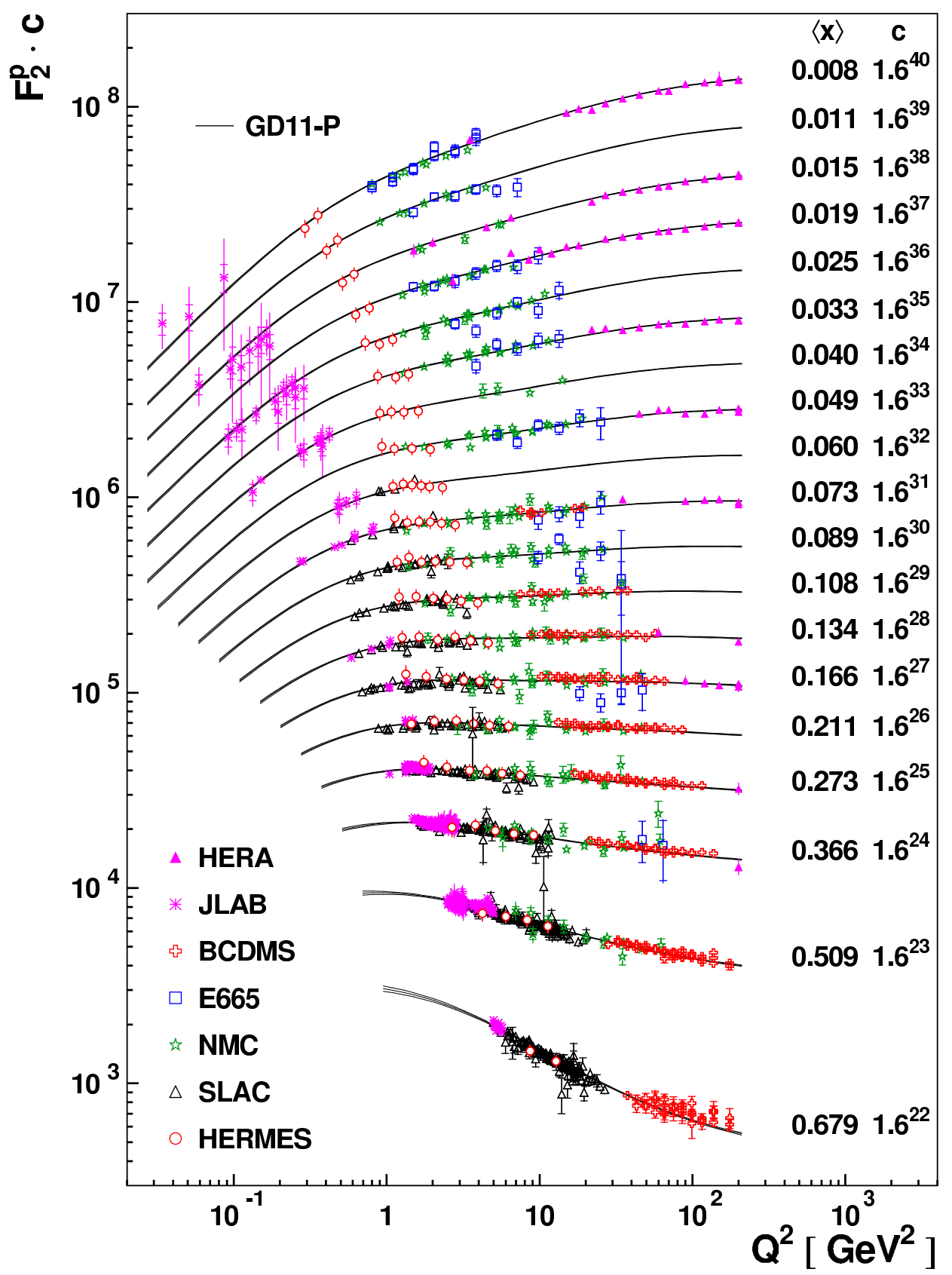

Figure 7. HERMES data for $F_{2}^{p}$ together with world data in the kinematic range $0.008 \leq\langle x\rangle \leq$ 0.679 and $0.02 \mathrm{GeV}^{2} \leq\left\langle Q^{2}\right\rangle \leq 20 \mathrm{GeV}^{2}$. The results are overlaid with the phenomenological parameterization GD11-P (black solid central curve) and its uncertainty (outer curves) obtained as described in section 6.3. A bin-centering correction is applied to the data in order to match the central values of the $x$ bins. The values of $F_{2}^{p}$ are scaled by powers of 1.6. Inner error bars are statistical uncertainties, while outer error bars are total uncertainties calculated as the sum in quadrature of all statistical and systematic uncertainties including normalization. 


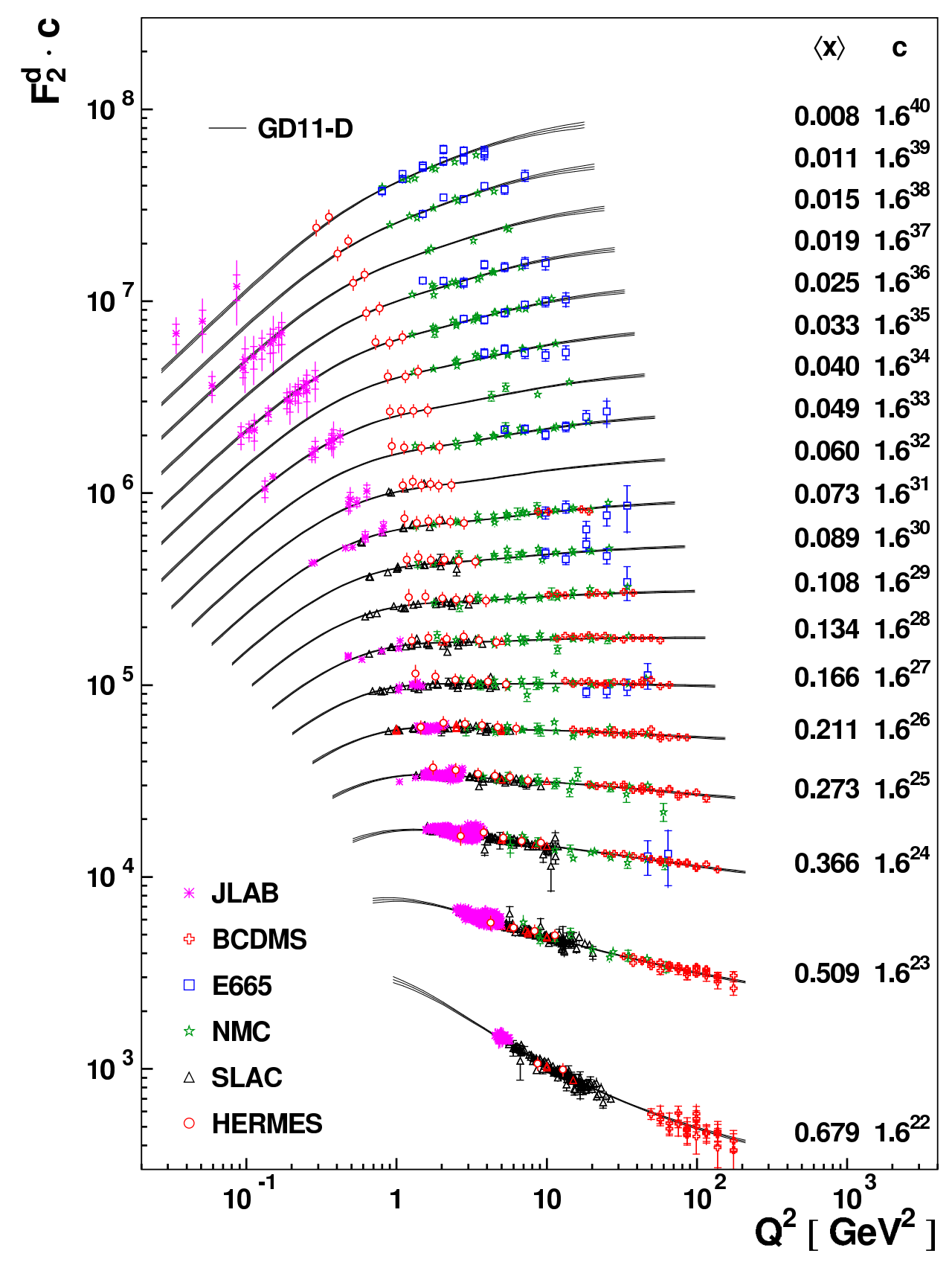

Figure 8. HERMES data for $F_{2}^{d}$ together with world data in the kinematic range $0.008 \leq\langle x\rangle \leq$ 0.679 and $0.02 \mathrm{GeV}^{2} \leq\left\langle Q^{2}\right\rangle \leq 20 \mathrm{GeV}^{2}$. The results are overlaid with the phenomenological parameterization GD11-D (black solid central curve) and its uncertainty (outer curves) obtained as described in section 6.3. A bin-centering correction is applied to the data in order to match the central values of the $x$ bins. The values of $F_{2}^{d}$ are scaled by powers of 1.6. Inner error bars are statistical uncertainties, while outer error bars are total uncertainties calculated as the sum in quadrature of all statistical and systematic uncertainties including normalization. 
For virtual-photon data $\left(Q^{2}>0\right)$, the photon-nucleon cross section $\sigma_{L+T}^{p, d}=\sigma_{L}^{p, d}+\sigma_{T}^{p, d}$ can be derived from the structure function $F_{2}^{p, d}$ using eq. (2.4):

$$
\sigma_{L+T}^{p, d}=4 \pi^{2} \alpha_{\mathrm{em}} \frac{Q^{2}+4 M^{2} x^{2}}{Q^{4}(1-x)} F_{2}^{p, d} .
$$

The $W^{2}$ dependence of the resulting proton and deuteron cross sections $\sigma_{L+T}^{p, d}$ is shown in figures 9 and 10 for HERMES results on inelastic scattering in comparison with world data and real-photon cross sections. HERMES data fill a gap for $Q^{2}$ in the range $0.3-1 . \mathrm{GeV}^{2}$ and $W^{2}$ in the range $20-50 \mathrm{GeV}^{2}$.

\subsection{Cross-section ratio $\sigma^{d} / \sigma^{p}$}

From the measured cross-section ratio, $\sigma^{d} / \sigma^{p}$, the ratio of the deuteron and proton structure functions, $F_{2}^{d} / F_{2}^{p}$, can be extracted. In the DIS regime $F_{2}^{d} / F_{2}^{p}$ is related to the ratio of the down and up quark distribution functions and imposes strong constraints on the $x$ dependence of the flavor composition of the nucleon. From the combination of $F_{2}^{p}$ and $F_{2}^{d} / F_{2}^{p}$ the non-singlet structure function $F_{2}^{p}-F_{2}^{n}$ and the Gottfried sum $S_{G}=\int\left(F_{2}^{p}-F_{2}^{n}\right) d x / x$ can be determined. For the latter, it was shown that the simple quark-model expectation of $1 / 3$ is not reached $[42,43]$ and hence the light quark sea is not flavor-symmetric. We do not elaborate on these aspects here, as our data add only limited additional information in the DIS region.

The cross-section ratio $\sigma^{d} / \sigma^{p}$ is determined year-by-year and then averaged. Thereby the normalization uncertainty is reduced and the effects of PID efficiencies and contaminations cancel. Because the deuteron and the proton data were partly taken with different beam conditions and resulting possible differences in absolute normalization, the ratio of the $F_{2}$-values given in tables 5 and 6 may differ slightly from the ratio values presented here. The results for $\sigma^{d} / \sigma^{p}$ are listed in table 7 . The values are shown as a function of $Q^{2}$ in bins of $x$ in figure 11 together with world data.

\subsection{Fits to world data to the cross section $\sigma_{L+T}^{p, d}$}

Fits of the inclusive inelastic scattering cross sections on the proton and deuteron are performed. The ALLM functional form selected for the fits is described in refs. [3] and [25]. It is a 23-parameter Regge-motivated model of $\sigma_{L+T}^{p, d}=\sigma_{L}^{p, d}+\sigma_{T}^{p, d}$, valid for $W^{2}>4 \mathrm{GeV}^{2}$, i.e., above the resonance region, and any $Q^{2}$ including the real photon point $\left(Q^{2}=0\right)$.

These new fits to the photon-nucleon cross sections $\sigma_{L+T}$ on proton (GD11-P) and deuteron (GD11-D) reflect the recent world knowlege on the cross sections. In order to present a self-consistent set, the values of $F_{2}$ to be used in the fit are calculated from the measured cross sections using for all data the parametrization $R_{1998}$. In cases where the measured cross sections were not given, the latter are reconstructed from the published values for $F_{2}$ using the values of $R$ used in calculating these $F_{2}$. Both fits GD11-P and GD11-D are performed in the same manner.

The fit to proton data includes 2441 data points from the SLAC experiments E49a, E49b, E61, E87, E89a, E89b [19], NMC [17], the combined HERA data [44], E665 [18], 


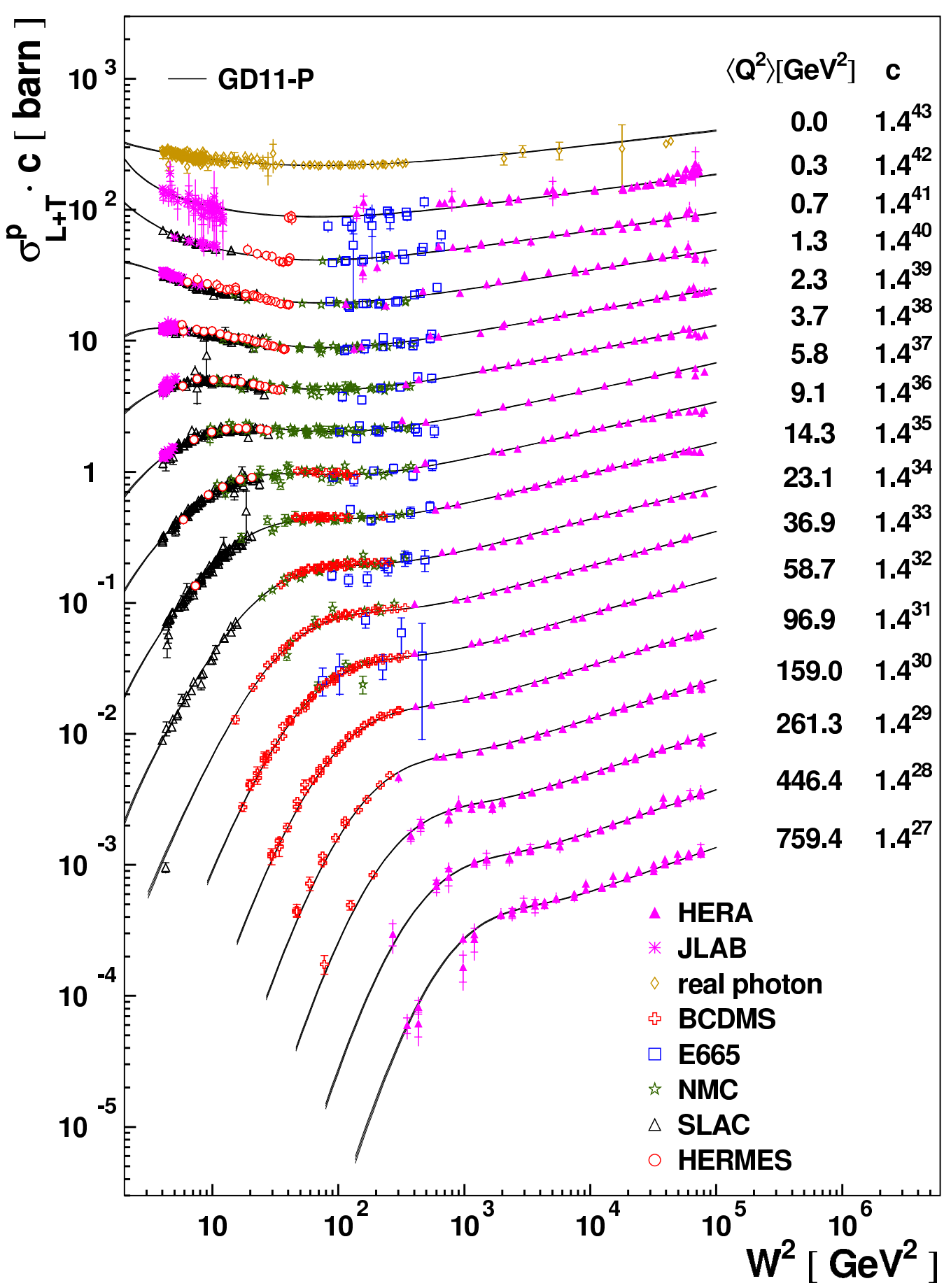

Figure 9. HERMES data for the photon-proton cross section $\sigma_{L+T}^{p}$ as a function of $W^{2}$, together with world data and the results from the GD11-P fit (central curves) and its uncertainties (outer curves), in bins of $Q^{2}$. The data points denoted 'real photon' are for photoproduction. Inner error bars are statistical uncertainties, while outer error bars are total uncertainties calculated as the sum in quadrature of all statistical and systematic uncertainties including normalization. 


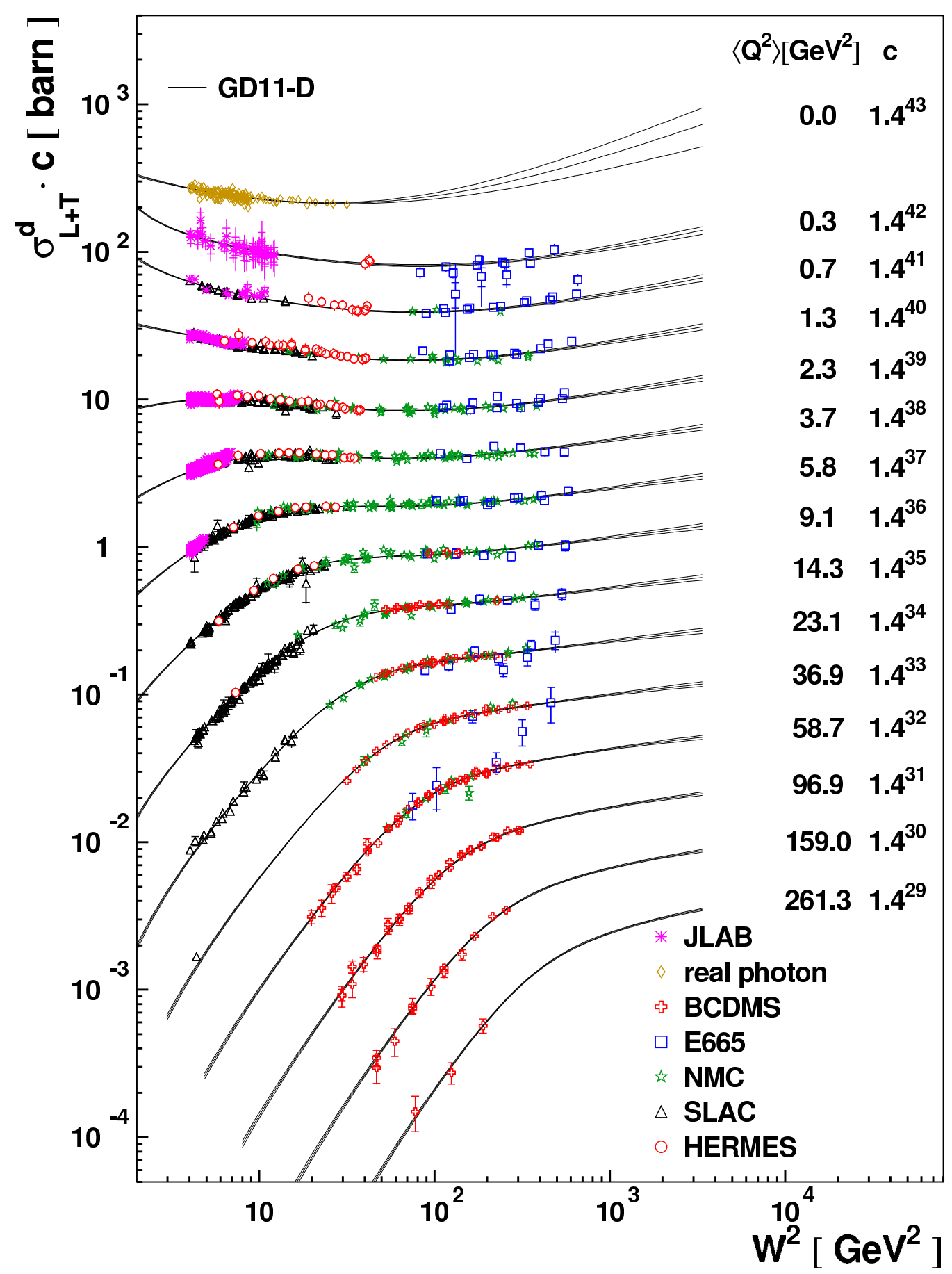

Figure 10. HERMES data for the photon-deuteron cross section $\sigma_{L+T}^{d}$ as a function of $W^{2}$, together with world data and the results from the GD11-D fit (central curves) and its uncertainties (outer curves), in bins of $Q^{2}$. The data points denoted 'real photon' are for photoproduction. Inner error bars are statistical uncertainties, while outer error bars are total uncertainties calculated as the sum in quadrature of all statistical and systematic uncertainties including normalization. 


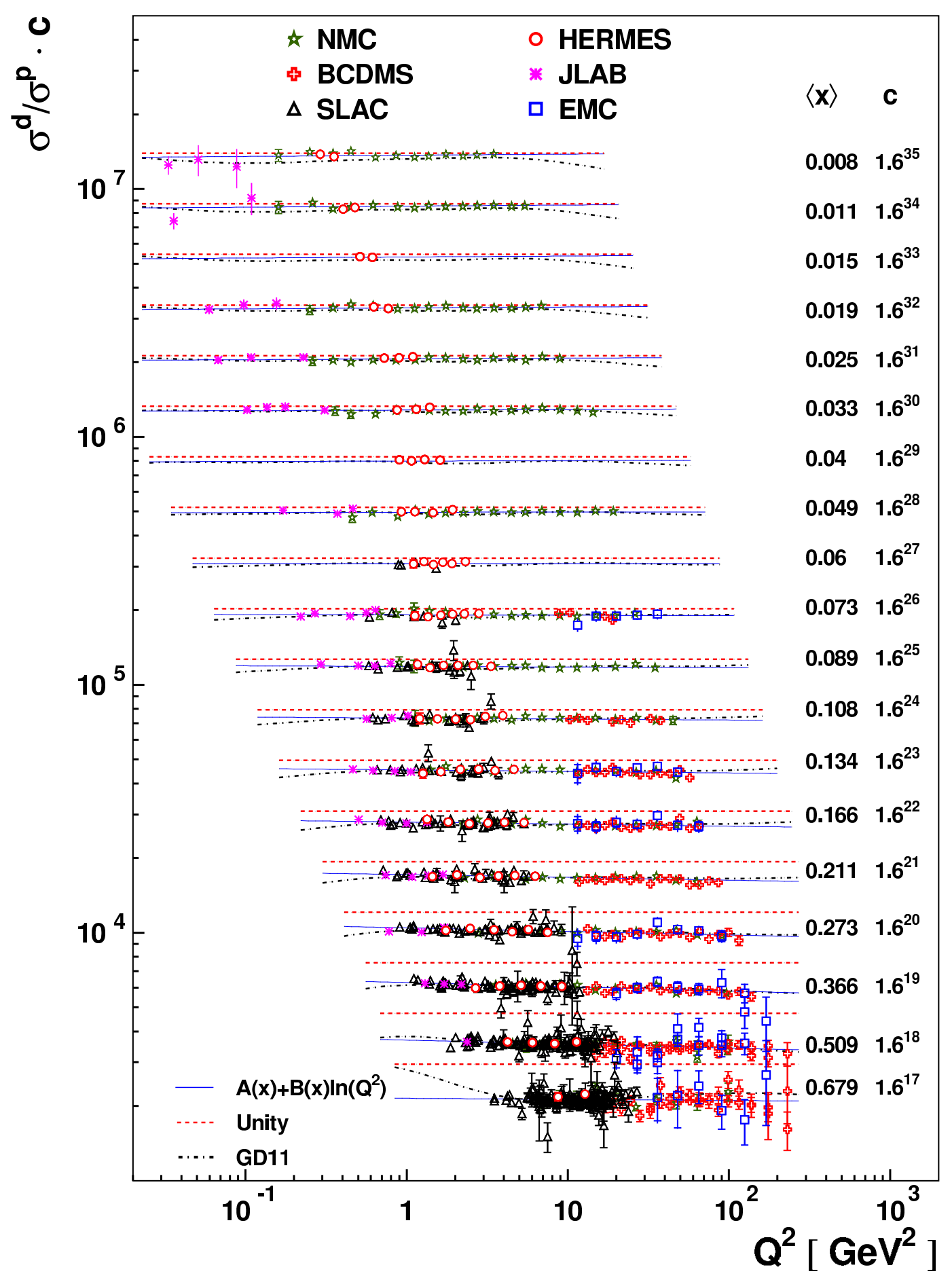

Figure 11. The $Q^{2}$ dependence of $\sigma^{d} / \sigma^{p}$ for data from SLAC, HERMES, EMC, NMC, JLAB and BCDMS in bins of $\langle x\rangle$. The error bars on the data points represent total uncertainties. The data points are overlaid with the results from the fit described in section 6.2 (blue solid line), with the unity line (red dashed) and with the ratio of the GD11-D over GD11-P fits (green dash-dotted line). 


\begin{tabular}{|l|c|c|c|c|}
\hline Experiment & $\begin{array}{c}\# \text { of } \\
\text { points }\end{array}$ & $\langle x\rangle$ range & $\begin{array}{c}\left\langle Q^{2}\right\rangle \text { range } \\
{\left[\mathrm{GeV}^{2}\right]}\end{array}$ & target \\
\hline HERA (positron beam) [44] & 456 & $0.621 \times 10^{-6}-0.65$ & $0.045-1000$ & $\mathrm{p}$ \\
HERA (electron beam) [44] & 95 & $0.13 \times 10^{-2}-0.65$ & $90-1000$ & $\mathrm{p}$ \\
E665 [18] & 91 & $0.89 \times 10^{-3}-0.39$ & $0.23-64$ & $\mathrm{p}, \mathrm{d}$ \\
NMC - 90 GeV [17] & 73 & $0.78 \times 10^{-2}-0.465$ & $0.8-8.75$ & $\mathrm{p}, \mathrm{d}$ \\
NMC - 120 GeV [17] & 65 & $0.87 \times 10^{-2}-0.475$ & $1.18-14.4$ & $\mathrm{p}, \mathrm{d}$ \\
NMC - 200 GeV [17] & 75 & $0.35 \times 10^{-2}-0.477$ & $0.83-34.8$ & $\mathrm{p}, \mathrm{d}$ \\
NMC - 280 GeV [17] & 79 & $0.37 \times 10^{-2}-0.479$ & $1.27-62.3$ & $\mathrm{p}, \mathrm{d}$ \\
BCDMS - 100 GeV [15] & 58 & $0.07-0.75$ & $7.5-75$ & $\mathrm{p}$ \\
BCDMS - 120 GeV [15] & 62 & $0.07-0.75$ & $8.75-99$ & $\mathrm{p}, \mathrm{d}$ \\
BCDMS - 200 GeV [15] & 57 & $0.07-0.75$ & $8.75-137$ & $\mathrm{p}$ \\
BCDMS - 200 GeV [15] & 56 & $0.07-0.75$ & $17-137.5$ & $\mathrm{~d}$ \\
BCDMS - 280 GeV [15] & 52 & $0.1-0.75$ & $32.5-230$ & $\mathrm{p}, \mathrm{d}$ \\
SLAC E49a [19] & 98 & $0.067-0.71$ & $0.59-7.9$ & $\mathrm{p}, \mathrm{d}$ \\
SLAC E49b [19] & 187 & $0.08-0.854$ & $0.663-20$ & $\mathrm{p}$ \\
SLAC E49b [19] & 174 & $0.08-0.854$ & $0.663-20$ & $\mathrm{~d}$ \\
SLAC E61 [19] & 25 & $0.065-0.326$ & $0.58-1.68$ & $\mathrm{p}$ \\
SLAC E61 [19] & 24 & $0.065-0.326$ & $0.58-1.68$ & $\mathrm{~d}$ \\
SLAC E87 [19] & 94 & $0.313-0.832$ & $3.96-19.7$ & $\mathrm{p}, \mathrm{d}$ \\
SLAC E89a [19] & 72 & $0.31-0.9$ & $3.6-30.3$ & $\mathrm{p}$ \\
SLAC E89a [19] & 66 & $0.33-0.9$ & $3.86-30.2$ & $\mathrm{~d}$ \\
SLAC E89b [19] & 98 & $0.063-0.809$ & $0.887-18.4$ & $\mathrm{p}$ \\
SLAC E89b [19] & 81 & $0.063-0.807$ & $0.887-18.4$ & $\mathrm{~d}$ \\
SLAC E139 [19] & 18 & $0.089-0.7$ & $2-15$ & $\mathrm{~d}$ \\
SLAC E140 [19] & 38 & $0.2-0.5$ & $1-10$ & $\mathrm{~d}$ \\
JLAB E00-116 [22] & 50 & $0.483-0.641$ & $3.585-5.67$ & $\mathrm{p}, \mathrm{d}$ \\
JLAB CLAS [20] & 272 & $0.23-0.509$ & $1.325-3.275$ & $\mathrm{p}$ \\
JLAB CLAS [21] & 5 & $0.122-0.617$ & $0.475-5.125$ & $\mathrm{~d}$ \\
JLAB HALL C (Rosenbluth) [23] & 50.8 & $0.15-1.045$ & $\mathrm{p}, \mathrm{d}$ \\
JLAB HALL C (Model Dep.) [23] & $50.9 \times 10^{-2}-0.25$ & $0.034-1.761$ & $\mathrm{p}, \mathrm{d}$ \\
HERMES (this analysis) & 196 & - & - & $\mathrm{p}, \mathrm{d}$ \\
real photon [45] & 174 & $-69 \times 10^{-2}-0.664$ & $0.291-12.78$ & - \\
real photon [45] & & & $\mathrm{d}$ \\
\hline
\end{tabular}

Table 3. Data sets used in the GD11 fits.

BCDMS [15], JLAB [20, 22, 23], the HERMES data presented here, and real photon data [45]. In the case of HERA, only data points with $Q^{2}<1000 \mathrm{GeV}^{2}$ were used in order to minimize contributions from weak processes to the cross section. The fit to deuteron data includes 2497 data points from the SLAC experiments E49a, E49b, E61, E87, E89a, E89b, E139, E140 [19], NMC [17]; E665 [18]; BCDMS [15]; JLAB [21-23]; the HERMES data presented in this paper, and real photon data [45]. Table 3 lists in more detail the data sets, together with their $\langle x\rangle$ and $\left\langle Q^{2}\right\rangle$ ranges. 
The fits are based on the minimization of the value of $\chi^{2}$ defined as

$$
\begin{aligned}
\chi^{2}(\mathbf{p}, \boldsymbol{\nu}) & =\sum_{i, k} \frac{\left[D_{i, k}\left(W^{2}, Q^{2}\right) \cdot\left(1+\delta_{k} \nu_{k}\right)-T\left(\mathbf{p}, W^{2}, Q^{2}\right)\right]^{2}}{\left(\sigma_{i, k}^{\mathrm{stat}}{ }^{2}+\sigma_{i, k}^{\mathrm{syst}^{2}}\right) \cdot\left(1+\delta_{k} \nu_{k}\right)^{2}}+\sum_{k} \nu_{k}^{2} \\
& \approx \sum_{i, k} \frac{\left[D_{i, k}\left(W^{2}, Q^{2}\right)-T\left(\mathbf{p}, W^{2}, Q^{2}\right) \cdot\left(1-\delta_{k} \nu_{k}\right)\right]^{2}}{\sigma_{i, k}^{\mathrm{stat}^{2}}+\sigma_{i, k}^{\mathrm{syst}} 2}+\sum_{k} \nu_{k}^{2},
\end{aligned}
$$

where $D_{i, k} \pm \sigma_{i, k}^{\text {stat }} \pm \sigma_{i, k}^{\text {syst }}$ are the values of $\sigma_{L+T}$ for data point $i$ within the data set $k, \delta_{k}$ is the normalization uncertainty in data set $k$ quoted by the experiment, $\nu_{k}$ is a normalization parameter, $T\left(\mathbf{p}, W^{2}, Q^{2}\right)$ is the 23-parameter ALLM functional form, $\mathbf{p}$ is the vector of functional parameters and $\boldsymbol{\nu}$ is the vector of normalization parameters. The definition of $\chi^{2}$ takes into account point-by-point statistical and systematic uncertainties. We agree that ideally one should treat the statistical and systematic uncertainties separately, as the latter in most cases are to some degree correlated. However, almost all published data sets just give statistical, total, and normalization uncertainties. For that reason we have used in the fit the total uncertainties per point, plus the overall normalization. Clearly the value of $\chi^{2}$ is influenced by this, but in practice the influence on the resultant parameters seems minor, see for example ref. [46]. Additionally, for each data set $k$ a normalization parameter $\nu_{k}$ is used to describe the normalization of all data points belonging to the data set. Since the normalization parameters are assumed to normalize data within the known normalization uncertainties, they are scaled with these uncertainties and taken into account by a penalty term $\sum_{k} \nu_{k}^{2}$ to control their variation according to their standard deviations.

At each iteration of the $\chi^{2}$-minimization, the normalization parameters $\nu_{k}$ are analytically determined:

$$
\nu_{k}=\frac{\sum_{i} \delta_{k} T_{i, k}\left(T_{i, k}-D_{i, k}\right) / \sigma_{i, k}^{2}}{\sum_{i} T_{i, k}^{2} \delta_{k}^{2} / \sigma_{i, k}^{2}+1},
$$

where $\sigma_{i, k}^{2}=\sigma_{i, k}^{\text {stat }}{ }^{2}+\sigma_{i, k}^{\text {syst }}{ }^{2}$. This equation is obtained by requiring $\partial \chi^{2} / \partial \nu_{k}=0$ in the context of the approximation for $\chi^{2}$ in eq. (6.2). The analytical determination of the parameters $\nu_{k}$ reduces the number of free parameters in the $\chi^{2}$-minimization. The separate extraction of all normalization parameters is possible only because the parameters $\nu_{k}$ are uncorrelated. At each step eq. (6.3) is substituted into the $\chi^{2}$-function, eq. (6.2), and thus the final fit result is obtained by minimizing $\chi^{2}$ only with respect to the functional parameters. Similar methods are used by others in order to save computing time $[47,48]$.

The fits are performed using the CERNLIB package MINUIT [49] and the MINUIT extension [50]. The resulting fit parameters are listed in table 8 together with the parameters obtained in the previous fits ALLM97 [25] and GD07-P [51] performed on proton data. The difference between GD07-P and GD11-P is the presence of HERMES, JLAB and the combined HERA data in the most recent fit. In ref. [51] the uncertainties were calculated with an UP value [49] equal to 24.7. That identical fit was redone here with the same value of $\mathrm{UP}=1$ as the new fits in order to provide an appropriate comparison of its uncertainties. The ALLM97 fit provided no uncertainties. The uncertainties given in the table for GD07-P, GD11-P, and GD11-D correspond to the diagonal elements of the full covariance 


\begin{tabular}{|c|c|c|}
\hline parameter & value & uncertainty \\
\hline$p_{1}$ & 0.986 & 0.0021 \\
$p_{2}$ & -0.684 & 0.034 \\
$p_{3}$ & 1.54 & 0.18 \\
$p_{4}$ & -2.93 & 0.36 \\
$p_{5}$ & 1.96 & 0.22 \\
$p_{6}$ & 0.0053 & 0.0011 \\
$p_{7}$ & -0.0974 & 0.0087 \\
$p_{8}$ & 0.125 & 0.014 \\
\hline normalization & value & \\
\hline HERMES & 0.996 & - \\
NMC & 0.999 & - \\
BCDMS & 1.010 & - \\
SLAC & 1.003 & - \\
JLAB & 1.000 & - \\
EMC & 0.995 & - \\
\hline
\end{tabular}

Table 4. Results of fit to the cross-section ratio $\sigma^{d} / \sigma^{p}$. The meaning of the parameters $p_{1}, \ldots p_{8}$ and relative normalizations is explained in the text.

matrix, which must be used to calculate uncertainties in $F_{2}$ or cross sections. The $\chi^{2} /$ per degree of freedom for the GD11-P is 0.89 (it is 0.92 for GD07-P), while for the deuteron fit GD11-D it is 0.68. The fitted relative normalization of HERMES proton (deuteron) data is $+1.5 \%(-2.2 \%)$, well within the quoted uncertainty of $7.5 \%$ (7.6\%). Table 9 shows the fitted relative normalizations for all the data sets used in the fit.

\subsection{Fits to world data for the cross-section ratio $\sigma^{d} / \sigma^{p}$}

The world data on the cross-section ratio $\sigma^{d} / \sigma^{p}$ is fitted using the functional form

$$
\sigma^{d} / \sigma^{p}\left(x, Q^{2}\right)=A(x)+B(x) \ln Q^{2},
$$

with

$$
\begin{aligned}
& A(x)=p_{1}+p_{2} x+p_{3} x^{2}+p_{4} x^{3}+p_{5} x^{4}, \\
& B(x)=p_{6}+p_{7} x+p_{8} x^{2},
\end{aligned}
$$

previously used by NMC in ref. [34]. It includes eight free parameters $p_{1}, \ldots, p_{8}$. The full data set consists of 260 data points from NMC [52], 621 data points from SLAC [19], 49 data points from JLAB [23], 81 data points from this analysis, 159 data points from BCDMS, and 53 data points from EMC. The latter two data sets are taken from ref. [19]. The relative normalizations are also fitted using the same method as described in the previous section. The final results are listed in table 4 . The relative normalizations were all found to be well within the normalization uncertainties quoted by the experiments. In the case of HERMES data, it is found to be 0.996. Figure 11 shows the world data of 
$\sigma^{d} / \sigma^{p}$ overlapped with the fits described in this section and with the ratio of $F_{2}^{d}$ to $F_{2}^{p}$ derived from the GD11 fits. While the ratio deviates from unity at large $x$, both GD11 and NMC-like fits describe the data well but start to deviate from each other outside the range where the ratio has been determined directly.

\section{Summary}

This high-statistics measurement of the inelastic scattering cross section on the proton and the deuteron by HERMES provides data in the ranges $0.006 \leq x \leq 0.9$ and $0.1 \mathrm{GeV}^{2} \leq$ $Q^{2} \leq 20 \mathrm{GeV}^{2}$ and contributes data in a kinematic region previously not covered, $Q^{2} \lesssim$ $1 \mathrm{GeV}^{2}$ and $15 \mathrm{GeV}^{2}<W^{2}<45 \mathrm{GeV}^{2}$, corresponding to $0.006<x<0.04$. In the region of overlap, HERMES data agree with the world data. The new results are fitted in conjunction with the other data, including those at the photon point, to give a new parameterization of the structure functions $F_{2}^{p}\left(F_{2}^{d}\right)$ for $Q^{2}=0$ to $30000 \mathrm{GeV}^{2}\left(230 \mathrm{GeV}^{2}\right)$ and $x$-values $0.6 \times 10^{-6}\left(0.89 \times 10^{-3}\right)$ to 0.9 . The fitted relative normalizations of HERMES data are well within the quoted normalization uncertainties.

The proton and deuteron cross-section ratio was also determined. This quantity has a much smaller point-to-point uncertainty because several systematic uncertainties cancel in the ratio and its normalization uncertainty is also much smaller. The HERMES data show excellent agreement with the other data and the fitted normalization is well within the quoted normalization uncertainty. The cross-section ratio from HERMES is also fitted in conjunction with existing data from other experiments. The $Q^{2}$ dependence can be well described by a simple (x-dependent) $\ln Q^{2}$ term.

\section{Acknowledgments}

We gratefully acknowledge the DESY management for its support and the staff at DESY and the collaborating institutions for their significant effort. This work was supported by the Ministry of Economy and the Ministry of Education and Science of Armenia; the FWO-Flanders and IWT, Belgium; the Natural Sciences and Engineering Research Council of Canada; the National Natural Science Foundation of China; the Alexander von Humboldt Stiftung, the German Bundesministerium für Bildung und Forschung (BMBF), and the Deutsche Forschungsgemeinschaft (DFG); the Italian Istituto Nazionale di Fisica Nucleare (INFN); the MEXT, JSPS, and G-COE of Japan; the Dutch Foundation for Fundamenteel Onderzoek der Materie (FOM); the Russian Academy of Science and the Russian Federal Agency for Science and Innovations; the U.K. Engineering and Physical Sciences Research Council, the Science and Technology Facilities Council, and the Scottish Universities Physics Alliance; the U.S. Department of Energy (DOE) and the National Science Foundation (NSF); the Basque Foundation for Science (IKERBASQUE); and the European Community Research Infrastructure Integrating Activity under the FP7 "Study of Strongly Interacting Matter (HadronPhysics2, Grant Agreement number 227431)". 


\section{A Tables}

\begin{tabular}{|c|c|c|c|c|c|c|c|c|c|c|}
\hline$x$ bin & $Q^{2}$ bin & $\langle x\rangle$ & $\begin{array}{c}\left\langle Q^{2}\right\rangle \\
{\left[\mathrm{GeV}^{2}\right]}\end{array}$ & $\begin{array}{c}\frac{d^{2} \sigma^{p}}{d x d Q^{2}} \\
{\left[\mathrm{nb} / \mathrm{GeV}^{2}\right]}\end{array}$ & $F_{2}^{p}$ & $\begin{array}{r}\delta_{\text {stat. }} \\
{[\%]}\end{array}$ & $\begin{array}{r}\delta_{\mathrm{PID}} \\
{[\%]}\end{array}$ & $\begin{array}{r}\delta_{\text {model }} \\
{[\%]}\end{array}$ & $\begin{array}{r}\delta_{\text {mis. }} \\
{[\%]}\end{array}$ & $\begin{array}{r}\delta_{\text {rad. }} \\
{[\%]}\end{array}$ \\
\hline 1 & $\overline{\mathrm{A}}$ & 0.0069 & 0.291 & $0.323 \times 10^{5}$ & 0.162 & 1.60 & 0.69 & 4.30 & 5.36 & 1.75 \\
\hline 2 & A & 0.0102 & 0.404 & $0.143 \times 10^{5}$ & 0.200 & 1.35 & 0.87 & 2.79 & 3.95 & 0.48 \\
\hline 3 & A & 0.0142 & 0.514 & $0.732 \times 10^{4}$ & 0.219 & 0.91 & 0.61 & 2.22 & 4.36 & 0.08 \\
\hline 4 & A & 0.0186 & 0.626 & $0.432 \times 10^{4}$ & 0.241 & 0.99 & 0.36 & 1.85 & 3.89 & 0.10 \\
\hline 5 & A & 0.0244 & 0.725 & $0.307 \times 10^{4}$ & 0.277 & 0.83 & 0.10 & 1.66 & 4.66 & 0.24 \\
\hline 6 & A & 0.0325 & 0.872 & $0.182 \times 10^{4}$ & 0.299 & 0.88 & 0.01 & 1.54 & 3.78 & 0.39 \\
\hline 7 & A & 0.0398 & 0.903 & $0.157 \times 10^{4}$ & 0.310 & 1.32 & 0.05 & 1.71 & 3.92 & 0.71 \\
\hline 8 & A & 0.0486 & 0.929 & $0.141 \times 10^{4}$ & 0.334 & 2.15 & 0.07 & 2.76 & 6.43 & 0.27 \\
\hline 9 & A & 0.0595 & 1.102 & $0.830 \times 10^{3}$ & 0.334 & 2.44 & 0.05 & 1.41 & 3.17 & 0.60 \\
\hline 10 & A & 0.0724 & 1.127 & $0.764 \times 10^{3}$ & 0.368 & 2.39 & 0.06 & 1.20 & 5.49 & 0.71 \\
\hline 11 & A & 0.0882 & 1.167 & $0.585 \times 10^{3}$ & 0.351 & 2.25 & 0.07 & 0.94 & 3.45 & 0.67 \\
\hline 12 & A & 0.1078 & 1.205 & $0.498 \times 10^{3}$ & 0.373 & 3.23 & 0.07 & 0.85 & 5.93 & 0.54 \\
\hline 13 & A & 0.1330 & 1.263 & $0.375 \times 10^{3}$ & 0.369 & 2.58 & 0.07 & 0.92 & 2.56 & 0.83 \\
\hline 14 & A & 0.1657 & 1.337 & $0.287 \times 10^{3}$ & 0.383 & 2.25 & 0.06 & 1.15 & 7.52 & 1.27 \\
\hline 15 & A & 0.2093 & 1.447 & $0.177 \times 10^{3}$ & 0.341 & 1.75 & 0.06 & 1.92 & 2.99 & 0.62 \\
\hline 16 & A & 0.2642 & 1.749 & $0.997 \times 10^{2}$ & 0.352 & 1.10 & 0.05 & 2.87 & 3.29 & 0.55 \\
\hline 17 & A & 0.3477 & 2.672 & $0.244 \times 10^{2}$ & 0.269 & 1.22 & 0.05 & 2.15 & 7.62 & 0.15 \\
\hline 18 & A & 0.4605 & 4.224 & $0.475 \times 10$ & 0.179 & 1.09 & 0.04 & 1.83 & 4.10 & 0.08 \\
\hline 19 & A & 0.6346 & 8.688 & 0.266 & 0.064 & 2.62 & 0.08 & 1.31 & 1.17 & 0.06 \\
\hline 1 & B & 0.0085 & 0.354 & $0.205 \times 10^{5}$ & 0.191 & 1.45 & 0.93 & 3.31 & 3.75 & 1.35 \\
\hline 2 & B & 0.0117 & 0.477 & $0.986 \times 10^{4}$ & 0.228 & 0.85 & 1.22 & 2.77 & 2.07 & 1.44 \\
\hline 3 & B & 0.0152 & 0.612 & $0.492 \times 10^{4}$ & 0.243 & 0.68 & 1.50 & 2.53 & 1.03 & 0.76 \\
\hline 4 & B & 0.0193 & 0.770 & $0.262 \times 10^{4}$ & 0.261 & 0.70 & 1.80 & 2.27 & 0.79 & 0.51 \\
\hline 5 & B & 0.0250 & 0.896 & $0.169 \times 10^{4}$ & 0.272 & 0.56 & 1.07 & 1.83 & 0.56 & 0.41 \\
\hline 6 & B & 0.0327 & 1.144 & $0.878 \times 10^{3}$ & 0.295 & 0.71 & 0.92 & 1.71 & 0.64 & 0.15 \\
\hline 7 & B & 0.0401 & 1.074 & $0.103 \times 10^{4}$ & 0.315 & 1.09 & 0.01 & 1.46 & 2.61 & 0.15 \\
\hline 8 & B & 0.0490 & 1.123 & $0.862 \times 10^{3}$ & 0.326 & 1.06 & 0.03 & 1.37 & 2.47 & 0.23 \\
\hline 9 & B & 0.0596 & 1.281 & $0.591 \times 10^{3}$ & 0.343 & 2.00 & 0.06 & 0.92 & 2.26 & 0.56 \\
\hline 10 & B & 0.0725 & 1.359 & $0.466 \times 10^{3}$ & 0.349 & 1.72 & 0.06 & 0.65 & 3.96 & 0.40 \\
\hline 11 & B & 0.0884 & 1.399 & $0.407 \times 10^{3}$ & 0.371 & 1.78 & 0.06 & 0.59 & 3.58 & 0.14 \\
\hline
\end{tabular}

Table 5. Results on the differential Born cross section $\frac{d^{2} \sigma^{p}}{d x d Q^{2}}$ and $F_{2}^{p}$. The statistical uncertainty $\delta_{\text {stat. }}$ and the systematic uncertainties $\delta_{\text {PID }}$ (particle identification), $\delta_{\text {model }}$ (model dependence outside the acceptance), $\delta_{\text {mis. }}$ (misalignment), and $\delta_{\text {rad. }}$ (Bethe-Heitler efficiencies) are given in percent. Corresponding $x$ bin numbers and $Q^{2}$ bin numbers and the average values $\langle x\rangle$ and $\left\langle Q^{2}\right\rangle$ are listed in the first four columns. The overall normalization uncertainty is $7.6 \%$. The structure function $F_{2}^{p}$ is derived using the parameterization $R=R_{1998}$ [32]. 


\begin{tabular}{|c|c|c|c|c|c|c|c|c|c|c|}
\hline$x$ bin & $2^{2}$ bin & $\langle x\rangle$ & $\begin{array}{c}\left\langle Q^{2}\right\rangle \\
{\left[\mathrm{GeV}^{2}\right]}\end{array}$ & $\begin{array}{c}\frac{d^{2} \sigma^{p}}{d x d Q^{2}} \\
{\left[\mathrm{nb} / \mathrm{GeV}^{2}\right]}\end{array}$ & $F_{2}^{p}$ & $\begin{array}{r}\delta_{\text {stat. }} \\
{[\%]}\end{array}$ & $\begin{array}{r}\delta_{\mathrm{PID}} \\
{[\%]}\end{array}$ & $\begin{array}{r}\delta_{\text {model }} \\
{[\%]}\end{array}$ & $\begin{array}{r}\delta_{\text {mis. }} \\
{[\%]}\end{array}$ & $\begin{array}{r}\delta_{\text {rad. }} \\
{[\%]}\end{array}$ \\
\hline 12 & B & 0.1082 & 1.554 & $0.280 \times 10^{3}$ & 0.374 & 1.14 & 0.06 & 0.53 & 4.34 & 0.15 \\
\hline 13 & B & 333 & 1.632 & $0.215 \times 10^{3}$ & 0.372 & 1.67 & 0.06 & 0.56 & 1.84 & 0.35 \\
\hline 14 & B & 661 & 1 . & $0.144 \times 10^{3}$ & 373 & 0.97 & 0.05 & 0.57 & 4.10 & 0.14 \\
\hline 15 & B & 104 & 2.050 & $0.857 \times 10^{2}$ & 0.352 & 1.38 & 0.05 & 0.64 & 2.80 & 0.26 \\
\hline 16 & B & 722 & 2.471 & $0.432 \times 10^{2}$ & 0.329 & 0.95 & 0.05 & 0.85 & 3.85 & 0.17 \\
\hline 17 & B & 620 & 3.790 & $0.110 \times 10^{2}$ & 0.268 & 0.81 & 0.04 & 0.64 & 2.21 & 0.06 \\
\hline 18 & $\mathrm{~L}$ & 0 & 6.026 & $0.180 \times 10$ & 56 & 0.62 & 0.04 & 0.69 & 0.94 & 0.02 \\
\hline 19 & B & 641 & 12.778 & $0.795 \times 10^{-1}$ & 0.047 & 2.19 & 0.05 & 0.67 & 0.86 & 0.10 \\
\hline 5 & C & 270 & 1.092 & $0.102 \times 10^{4}$ & 0.289 & 0.76 & 2.39 & 2.20 & 1.06 & 0.18 \\
\hline 6 & C & 8 & & $0.531 \times 10^{3}$ & 307 & 1.08 & 2.98 & 2.32 & 0.28 & 0.29 \\
\hline 7 & C & 1 & 1.293 & $0.629 \times 10^{3}$ & 0.313 & 0.83 & 0.38 & 1.54 & 2.71 & 0.17 \\
\hline 8 & $\mathrm{C}$ & 0.0490 & 1.460 & $0.445 \times 10^{3}$ & 0.327 & 0.70 & 0.17 & 1.18 & 1.29 & 0.12 \\
\hline 9 & $\mathrm{C}$ & 6 & 1.466 & $0.419 \times 10^{3}$ & .339 & 1.10 & 0.01 & 0.80 & 1.73 & 0.45 \\
\hline 10 & $\mathrm{C}$ & 0.0726 & 1.627 & $0.305 \times 10^{3}$ & 0.353 & 1.08 & 0.04 & 0.56 & 2.05 & 0.25 \\
\hline 11 & $\mathrm{C}$ & 0 & 1 & $0.247 \times 10^{3}$ & 0.351 & 1.09 & 0.05 & 0.49 & 1.43 & 0.06 \\
\hline 12 & C & 2 & 2.013 & $0.151 \times 10^{3}$ & 0.367 & 0.95 & 0.05 & 0.41 & 2.70 & 0.06 \\
\hline 13 & C & 0 & 2.1 & $0.109 \times 10^{3}$ & 0.359 & 1.04 & 0.05 & 0.39 & 2.17 & 0.15 \\
\hline 14 & C & 0.1662 & 2.4 & $0.703 \times 10^{2}$ & 0.363 & 1.15 & 0.05 & 0.35 & 0.98 & 0.12 \\
\hline 15 & C & 105 & 2.851 & $0.415 \times 10^{2}$ & 0.0 & 0.73 & 0.04 & 0.33 & 2.40 & 0.06 \\
\hline 16 & $\mathrm{C}$ & 4 & 3.479 & $0.195 \times 10^{2}$ & 6 & 0.66 & 0.04 & 0.31 & 1.74 & 0.09 \\
\hline 17 & C & & & $0.520 \times 10$ & & 0.60 & 0.04 & 0.23 & 1.84 & 0.02 \\
\hline 18 & C & & 8 . & 0.821 & $r$ & 0.66 & 0.05 & 0.23 & 2.23 & 0.00 \\
\hline 7 & 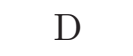 & 8 & 1 . & $0.349 \times 10^{3}$ & .317 & 0.72 & 2.58 & 2.08 & 0.53 & 0.35 \\
\hline 8 & D & & 1.92 & $0.210 \times 10^{3}$ & 0.323 & 0.65 & 2.49 & 1.74 & 0.68 & 0.20 \\
\hline 9 & $\mathrm{D}$ & & 1.6 & $0.297 \times 10^{3}$ & 0.337 & 1.22 & 0.04 & 0.79 & 1.88 & 0.18 \\
\hline 10 & D & 0 & 1.92 & $0.199 \times 10^{3}$ & 0.352 & 1.42 & 0.01 & 0.55 & 2.13 & 0.16 \\
\hline 11 & $\mathrm{D}$ & 0 & 2 . & $0.150 \times 10^{3}$ & 0.354 & 0.91 & 0.03 & 0.47 & 1.21 & 0.16 \\
\hline 12 & D & & 2 . & $0.893 \times 10^{2}$ & 0.364 & 0.99 & 0.04 & 0.37 & 1.50 & 0.13 \\
\hline 13 & $\mathrm{D}$ & & & & 0.372 & 0.82 & 0.04 & 0.31 & 2.67 & 0.10 \\
\hline 14 & D & & & $0.377 \times 10^{2}$ & 0.358 & 0.88 & 0.04 & 0.27 & 1.91 & 0.08 \\
\hline 15 & D & & & $0.219 \times 10^{2}$ & 0.343 & 0.68 & 0.05 & 0.22 & 1.80 & 0.09 \\
\hline 16 & D & & & $0.106 \times 10^{2}$ & 0.315 & 0.84 & 0.05 & 0.19 & 2.14 & 0.02 \\
\hline 17 & $\mathrm{D}$ & & & $0.263 \times 10$ & 0.242 & 0.57 & 0.04 & 0.13 & 1.63 & 0.01 \\
\hline 18 & $\mathrm{D}$ & 0.5038 & 11.344 & 0.350 & 0.132 & 0.98 & 0.07 & 0.32 & 1.61 & 0.01 \\
\hline 9 & $\mathrm{~F}$ & 0.0597 & 1.906 & $0.210 \times 10^{3}$ & 0.333 & 0.93 & 0.30 & 0.87 & 0.78 & 0.17 \\
\hline 10 & $\mathrm{E}$ & 0.0726 & 2.276 & $0.128 \times 10^{3}$ & 0.346 & 0.93 & 0.22 & 0.62 & 1.53 & 0.16 \\
\hline 11 & $\mathrm{E}$ & 0 . & 2. & $0.863 \times 10^{2}$ & 0.351 & 0.70 & 0.09 & 0.47 & 1.33 & 0.03 \\
\hline 12 & $\mathrm{E}$ & 0.1083 & 3.071 & $0.519 \times 10^{2}$ & 0.355 & 0.58 & 0.03 & 0.37 & 1.49 & 0.01 \\
\hline
\end{tabular}

Table 5. - continued. 


\begin{tabular}{|c|c|c|c|c|c|c|c|c|c|c|}
\hline$x$ bin & $Q^{2}$ bin & $\langle x\rangle$ & $\begin{array}{c}\left\langle Q^{2}\right\rangle \\
{\left[\mathrm{GeV}^{2}\right]}\end{array}$ & $\begin{array}{c}\frac{d^{2} \sigma^{p}}{d x d Q^{2}} \\
{\left[\mathrm{nb}^{\left.-\mathrm{GeV}^{2}\right]}\right.}\end{array}$ & $F_{2}^{p}$ & $\begin{array}{r}\delta_{\text {stat. }} \\
{[\%]}\end{array}$ & $\begin{array}{r}\delta_{\text {PID }} \\
{[\%]}\end{array}$ & $\begin{array}{r}\delta_{\text {model }} \\
{[\%]}\end{array}$ & $\begin{array}{r}\delta_{\text {mis. }} \\
{[\%]}\end{array}$ & $\begin{array}{r}\delta_{\text {rad. }} \\
{[\%]}\end{array}$ \\
\hline 13 & $\mathrm{E}$ & 0.1335 & 3.538 & $0.331 \times 10^{2}$ & 0.356 & 0.82 & 0.02 & 0.29 & 0.91 & 0.01 \\
14 & $\mathrm{E}$ & 0.1664 & 4.051 & $0.210 \times 10^{2}$ & 0.354 & 0.56 & 0.04 & 0.23 & 1.32 & 0.04 \\
15 & $\mathrm{E}$ & 0.2107 & 4.702 & $0.122 \times 10^{2}$ & 0.336 & 0.58 & 0.05 & 0.18 & 1.02 & 0.05 \\
16 & $\mathrm{E}$ & 0.2726 & 5.685 & $0.603 \times 10$ & 0.304 & 0.66 & 0.04 & 0.14 & 2.03 & 0.03 \\
17 & $\mathrm{E}$ & 0.3679 & 9.154 & $0.124 \times 10$ & 0.234 & 0.68 & 0.05 & 0.27 & 1.40 & 0.01 \\
9 & $\mathrm{~F}$ & 0.0603 & 2.319 & $0.124 \times 10^{3}$ & 0.330 & 0.76 & 2.40 & 1.35 & 0.77 & 0.13 \\
10 & $\mathrm{~F}$ & 0.0734 & 2.806 & $0.726 \times 10^{2}$ & 0.339 & 0.69 & 2.17 & 1.01 & 0.48 & 0.15 \\
11 & $\mathrm{~F}$ & 0.0894 & 3.341 & $0.444 \times 10^{2}$ & 0.349 & 0.68 & 1.58 & 0.77 & 0.66 & 0.11 \\
12 & $\mathrm{~F}$ & 0.1094 & 3.946 & $0.267 \times 10^{2}$ & 0.346 & 0.62 & 0.96 & 0.60 & 1.03 & 0.08 \\
13 & $\mathrm{~F}$ & 0.1347 & 4.618 & $0.164 \times 10^{2}$ & 0.346 & 0.58 & 0.41 & 0.46 & 0.56 & 0.03 \\
14 & $\mathrm{~F}$ & 0.1679 & 5.344 & $0.102 \times 10^{2}$ & 0.342 & 0.58 & 0.11 & 0.34 & 1.45 & 0.04 \\
15 & $\mathrm{~F}$ & 0.2128 & 6.266 & $0.592 \times 10$ & 0.330 & 0.63 & 0.02 & 0.27 & 2.05 & 0.02 \\
16 & $\mathrm{~F}$ & 0.2759 & 7.501 & $0.299 \times 10$ & 0.296 & 0.57 & 0.04 & 0.24 & 0.85 & 0.01 \\
\hline
\end{tabular}

Table 5. - continued.

\begin{tabular}{|c|c|c|c|c|c|r|r|r|r|r|}
\hline$x$ bin & $Q^{2}$ bin & $\langle x\rangle$ & $\begin{array}{c}\left\langle Q^{2}\right\rangle \\
{\left[\mathrm{GeV}^{2}\right]}\end{array}$ & $\begin{array}{c}\frac{d^{2} \sigma^{d}}{d x d Q^{2}} \\
{\left[\mathrm{nb}^{2} \mathrm{GeV}^{2}\right]}\end{array}$ & $\begin{array}{r}F_{2}^{d} \\
\delta_{\text {stat. }} \\
{[\%]}\end{array}$ & $\begin{array}{r}\delta_{\text {PID }} \\
{[\%]}\end{array}$ & $\begin{array}{r}\delta_{\text {model }} \\
{[\%]}\end{array}$ & $\begin{array}{r}\delta_{\text {mis. }} \\
{[\%]}\end{array}$ & $\begin{array}{r}\delta_{\text {rad. }} \\
{[\%]}\end{array}$ \\
\hline 1 & $\mathrm{~A}$ & 0.0069 & 0.291 & $0.327 \times 10^{5}$ & 0.164 & 1.41 & 0.69 & 3.14 & 5.36 & 0.36 \\
2 & $\mathrm{~A}$ & 0.0102 & 0.404 & $0.138 \times 10^{5}$ & 0.193 & 1.30 & 0.87 & 2.15 & 3.95 & 0.47 \\
3 & $\mathrm{~A}$ & 0.0142 & 0.514 & $0.724 \times 10^{4}$ & 0.217 & 0.84 & 0.61 & 1.70 & 4.36 & 0.40 \\
4 & $\mathrm{~A}$ & 0.0186 & 0.626 & $0.435 \times 10^{4}$ & 0.242 & 0.95 & 0.36 & 1.53 & 3.89 & 0.30 \\
5 & $\mathrm{~A}$ & 0.0244 & 0.725 & $0.304 \times 10^{4}$ & 0.274 & 0.81 & 0.10 & 1.43 & 4.66 & 0.27 \\
6 & $\mathrm{~A}$ & 0.0325 & 0.872 & $0.178 \times 10^{4}$ & 0.291 & 0.87 & 0.01 & 1.38 & 3.78 & 0.22 \\
7 & $\mathrm{~A}$ & 0.0398 & 0.903 & $0.155 \times 10^{4}$ & 0.306 & 1.32 & 0.05 & 1.50 & 3.92 & 0.10 \\
8 & $\mathrm{~A}$ & 0.0486 & 0.929 & $0.136 \times 10^{4}$ & 0.323 & 2.26 & 0.07 & 2.63 & 6.43 & 0.29 \\
9 & $\mathrm{~A}$ & 0.0595 & 1.102 & $0.803 \times 10^{3}$ & 0.323 & 2.60 & 0.05 & 1.33 & 3.17 & 0.70 \\
10 & $\mathrm{~A}$ & 0.0724 & 1.127 & $0.722 \times 10^{3}$ & 0.348 & 2.62 & 0.06 & 1.14 & 5.49 & 0.45 \\
11 & $\mathrm{~A}$ & 0.0882 & 1.167 & $0.564 \times 10^{3}$ & 0.338 & 2.53 & 0.07 & 0.84 & 3.45 & 1.18 \\
12 & $\mathrm{~A}$ & 0.1078 & 1.205 & $0.460 \times 10^{3}$ & 0.345 & 3.66 & 0.07 & 0.75 & 5.93 & 1.18 \\
13 & $\mathrm{~A}$ & 0.1330 & 1.263 & $0.335 \times 10^{3}$ & 0.329 & 3.06 & 0.07 & 0.78 & 2.56 & 0.94 \\
14 & $\mathrm{~A}$ & 0.1657 & 1.337 & $0.265 \times 10^{3}$ & 0.354 & 2.65 & 0.06 & 1.02 & 7.52 & 0.90 \\
15 & $\mathrm{~A}$ & 0.2093 & 1.447 & $0.155 \times 10^{3}$ & 0.298 & 2.21 & 0.06 & 1.87 & 2.99 & 0.52 \\
\hline
\end{tabular}

Table 6. Results on the differential Born cross section $\frac{d^{2} \sigma^{d}}{d x d Q^{2}}$ and $F_{2}^{d}$. The statistical uncertainty $\delta_{\text {stat. }}$ and the systematic uncertainties $\delta_{\text {PID }}$ (particle identification), $\delta_{\text {model }}$ (model dependence outside the acceptance), $\delta_{\text {mis. }}$ (misalignment), and $\delta_{\text {rad. }}$ (Bethe-Heitler efficiencies), are given in percent. Corresponding $x$ bin numbers and $Q^{2}$ bin numbers and the average values $\langle x\rangle$ and $\left\langle Q^{2}\right\rangle$ are listed in the first four columns. The overall normalization uncertainty is $7.5 \%$. The structure function $F_{2}^{d}$ is derived using the parameterization $R=R_{1998}[32]$. 


\begin{tabular}{|c|c|c|c|c|c|c|c|c|c|c|}
\hline$x$ bin & $Q^{2}$ bin & $\langle x\rangle$ & $\begin{array}{c}\left\langle Q^{2}\right\rangle \\
{\left[\mathrm{GeV}^{2}\right]}\end{array}$ & $\begin{array}{c}\frac{d^{2} \sigma^{d}}{d x d Q^{2}} \\
{\left[\mathrm{nb} / \mathrm{GeV}^{2}\right]}\end{array}$ & $F_{2}^{d}$ & $\begin{array}{r}\delta_{\text {stat. }} \\
{[\%]}\end{array}$ & $\begin{array}{r}\delta_{\mathrm{PID}} \\
{[\%]}\end{array}$ & $\begin{array}{r}\delta_{\text {model }} \\
{[\%]}\end{array}$ & $\begin{array}{r}\delta_{\text {mis. }} \\
{[\%]}\end{array}$ & $\begin{array}{r}\delta_{\text {rad. }} \\
{[\%]}\end{array}$ \\
\hline 16 & $\bar{A}$ & 2642 & 1.749 & $0.845 \times 10^{2}$ & 0.298 & 1.25 & 0.05 & 2.65 & 3.29 & 0.56 \\
\hline 17 & A & 3477 & 2.672 & $0.196 \times 10^{2}$ & 0.217 & 1.37 & 0.05 & 1.99 & 7.62 & 0.21 \\
\hline 18 & A & 4605 & 4.224 & $0.377 \times 10$ & 0.142 & 1.13 & 0.04 & 1.70 & 4.10 & 0.11 \\
\hline 19 & A & 5346 & 8.688 & 0.198 & 0.047 & 2.80 & 0.08 & 1.25 & 1.17 & 0.02 \\
\hline 1 & B & 0.0085 & 0.354 & $0.203 \times 10^{5}$ & 0.189 & 1.29 & 0.93 & 2.43 & 3.75 & 0.81 \\
\hline 2 & B & 0.0117 & 0.477 & $0.979 \times 10^{4}$ & 0.226 & 0.77 & 1.22 & 2.05 & 2.07 & 0.69 \\
\hline 3 & B & 0.0152 & 0.612 & $0.488 \times 10^{4}$ & 0.241 & 0.61 & 1.50 & 1.90 & 1.03 & 0.36 \\
\hline 4 & B & 0.0193 & 0.770 & $0.260 \times 10^{4}$ & 0.258 & 0.64 & 1.80 & 1.79 & 0.79 & 0.40 \\
\hline 5 & B & 0.0250 & 0.896 & $0.169 \times 10^{4}$ & 0.272 & 0.52 & 1.07 & 1.51 & 0.56 & 0.12 \\
\hline 6 & B & 0.0327 & 1.144 & $0.865 \times 10^{3}$ & 0.291 & 0.68 & 0.92 & 1.47 & 0.64 & 0.30 \\
\hline 7 & B & 0.0401 & 1.074 & $0.100 \times 10^{4}$ & 0.308 & 1.06 & 0.01 & 1.28 & 2.61 & 0.17 \\
\hline 8 & B & 0.0490 & 1.123 & $0.843 \times 10^{3}$ & 0.318 & 1.05 & 0.03 & 1.22 & 2.47 & 0.24 \\
\hline 9 & B & 0.0596 & 1.281 & $0.582 \times 10^{3}$ & 0.337 & 2.05 & 0.06 & 0.81 & 2.26 & 0.36 \\
\hline 10 & B & 0.0725 & 1.359 & $0.439 \times 10^{3}$ & 0.329 & 1.85 & 0.06 & 0.58 & 3.96 & 0.36 \\
\hline 11 & B & 0.0884 & 1.399 & $0.383 \times 10^{3}$ & 0.349 & 1.96 & 0.06 & 0.51 & 3.58 & 0.05 \\
\hline 12 & B & 0.1082 & 1.554 & $0.260 \times 10^{3}$ & 0.348 & 1.22 & 0.06 & 0.47 & 4.34 & 0.19 \\
\hline 13 & B & 0.1333 & 1.632 & $0.195 \times 10^{3}$ & 0.339 & 1.85 & 0.06 & 0.49 & 1.84 & 0.34 \\
\hline 14 & B & 0.1661 & 1.811 & $0.132 \times 10^{3}$ & 0.341 & 1.01 & 0.05 & 0.50 & 4.10 & 0.05 \\
\hline 15 & B & 0.2104 & 2.050 & $0.763 \times 10^{2}$ & 0.314 & 1.53 & 0.05 & 0.58 & 2.80 & 0.09 \\
\hline 16 & B & 0.2722 & 2.471 & $0.374 \times 10^{2}$ & 0.285 & 1.02 & 0.05 & 0.78 & 3.85 & 0.18 \\
\hline 17 & B & 0.3620 & 3.790 & $0.893 \times 10$ & 0.218 & 0.86 & 0.04 & 0.57 & 2.21 & 0.04 \\
\hline 18 & B & 0.4900 & 6.026 & $0.139 \times 10$ & 0.120 & 0.65 & 0.04 & 0.62 & 0.94 & 0.01 \\
\hline 19 & B & 0.6641 & 12.778 & $0.605 \times 10^{-1}$ & 0.036 & 2.32 & 0.05 & 0.66 & 0.86 & 0.01 \\
\hline 5 & $\mathrm{C}$ & 0.0270 & 1.092 & $0.103 \times 10^{4}$ & 0.292 & 0.68 & 2.39 & 1.81 & 1.06 & 0.25 \\
\hline 6 & $\mathrm{C}$ & 0.0338 & 1.391 & $0.535 \times 10^{3}$ & 0.309 & 0.99 & 2.98 & 1.93 & 0.28 & 0.43 \\
\hline 7 & $\mathrm{C}$ & 0.0401 & 1.293 & $0.621 \times 10^{3}$ & 0.309 & 0.80 & 0.38 & 1.35 & 2.71 & 0.15 \\
\hline 8 & $\mathrm{C}$ & 0.0490 & 1.460 & $0.430 \times 10^{3}$ & 0.316 & 0.70 & 0.17 & 1.05 & 1.29 & 0.12 \\
\hline 9 & $\mathrm{C}$ & 0.0596 & 1.466 & $0.400 \times 10^{3}$ & 0.323 & 1.09 & 0.01 & 0.69 & 1.73 & 0.24 \\
\hline 10 & $\mathrm{C}$ & 0.0726 & 1.627 & $0.291 \times 10^{3}$ & 0.337 & 1.10 & 0.04 & 0.48 & 2.05 & 0.07 \\
\hline 11 & $\mathrm{C}$ & 0.0885 & 1.691 & $0.236 \times 10^{3}$ & 0.337 & 1.11 & 0.05 & 0.41 & 1.43 & 0.02 \\
\hline 12 & $\mathrm{C}$ & 0.1082 & 2.013 & $0.140 \times 10^{3}$ & 0.341 & 0.96 & 0.05 & 0.37 & 2.70 & 0.08 \\
\hline 13 & $\mathrm{C}$ & 0.1334 & 2.158 & $0.102 \times 10^{3}$ & 0.335 & 1.09 & 0.05 & 0.33 & 2.17 & 0.05 \\
\hline 14 & $\mathrm{C}$ & 0.1662 & 2.458 & $0.636 \times 10^{2}$ & 0.328 & 1.22 & 0.05 & 0.30 & 0.98 & 0.07 \\
\hline 15 & $\mathrm{C}$ & 0.2105 & 2.851 & $0.361 \times 10^{2}$ & 0.310 & 0.75 & 0.04 & 0.29 & 2.40 & 0.07 \\
\hline 16 & $\mathrm{C}$ & 0.2724 & 3.479 & $0.167 \times 10^{2}$ & 0.272 & 0.67 & 0.04 & 0.28 & 1.74 & 0.03 \\
\hline 17 & C & 0.3622 & 5.125 & $0.428 \times 10$ & 0.205 & 0.62 & 0.04 & 0.21 & 1.84 & 0.04 \\
\hline 18 & $\tau$ & 0.4957 & 8.280 & 0.630 & 0.113 & 0.70 & 0.05 & 0.22 & 2.23 & 0.02 \\
\hline
\end{tabular}

Table 6. - continued. 


\begin{tabular}{|c|c|c|c|c|c|c|c|c|c|c|}
\hline$x$ bin & $Q^{2}$ bin & $\langle x\rangle$ & $\begin{array}{c}\left\langle Q^{2}\right\rangle \\
{\left[\mathrm{GeV}^{2}\right]}\end{array}$ & $\begin{array}{c}\frac{d^{2} \sigma^{d}}{d x d Q^{2}} \\
{\left[\mathrm{nb} / \mathrm{GeV}^{2}\right]}\end{array}$ & $F_{2}^{d}$ & $\begin{array}{r}\delta_{\text {stat. }} \\
{[\%]} \\
\end{array}$ & $\begin{array}{r}\delta_{\mathrm{PID}} \\
{[\%]}\end{array}$ & $\begin{array}{r}\delta_{\text {model }} \\
{[\%]}\end{array}$ & $\begin{array}{r}\delta_{\text {mis. }} \\
{[\%]}\end{array}$ & $\begin{array}{r}\delta_{\text {rad. }} \\
{[\%]}\end{array}$ \\
\hline 7 & $\mathrm{D}$ & 0.0408 & 1.615 & $0.343 \times 10^{3}$ & 0.311 & 0.68 & 2.58 & 1.76 & 0.53 & 0.34 \\
\hline 8 & $\mathrm{D}$ & 0.0496 & 1.927 & $0.208 \times 10^{3}$ & 0.320 & 0.60 & 2.49 & 1.49 & 0.68 & 0.10 \\
\hline 9 & $\mathrm{D}$ & 0.0596 & 1.675 & $0.290 \times 10^{3}$ & 0.328 & 1.21 & 0.04 & 0.69 & 1.88 & 0.06 \\
\hline 10 & $\mathrm{D}$ & 0.0726 & 1.928 & $0.192 \times 10^{3}$ & 0.339 & 1.46 & 0.01 & 0.46 & 2.13 & 0.01 \\
\hline 11 & $\mathrm{D}$ & 0.0885 & 2.080 & $0.144 \times 10^{3}$ & 0.339 & 0.89 & 0.03 & 0.37 & 1.21 & 0.07 \\
\hline 12 & $\mathrm{D}$ & 0.1083 & 2.490 & $0.824 \times 10^{2}$ & 0.336 & 1.01 & 0.04 & 0.31 & 1.50 & 0.18 \\
\hline 13 & $\mathrm{D}$ & 0.1334 & 2.795 & $0.570 \times 10^{2}$ & 0.345 & 0.83 & 0.04 & 0.26 & 2.67 & 0.11 \\
\hline 14 & $\mathrm{D}$ & 0.1663 & 3.195 & $0.343 \times 10^{2}$ & 0.326 & 0.90 & 0.04 & 0.22 & 1.91 & 0.01 \\
\hline 15 & D & 0.2106 & 3.709 & $0.195 \times 10^{2}$ & 0.305 & 0.69 & 0.05 & 0.19 & 1.80 & 0.04 \\
\hline 16 & $\mathrm{D}$ & 0.2726 & 4.527 & $0.899 \times 10$ & 0.266 & 0.88 & 0.05 & 0.17 & 2.14 & 0.04 \\
\hline 17 & $\mathrm{D}$ & 3624 & 6.797 & $0.215 \times 10$ & 0.197 & 0.58 & 0.04 & 0.12 & 1.63 & 0.01 \\
\hline 18 & $\mathrm{D}$ & 0.5038 & 11.344 & 0.272 & 0.103 & 1.03 & 0.07 & 0.31 & 1.61 & 0.02 \\
\hline 9 & $\mathrm{E}$ & 0.0597 & 1.906 & $0.202 \times 10^{3}$ & 0.321 & 0.90 & 0.30 & 0.74 & 0.78 & 0.08 \\
\hline 10 & $\mathrm{E}$ & 0.0726 & 2.276 & $0.124 \times 10^{3}$ & 0.334 & 0.92 & 0.22 & 0.53 & 1.53 & 0.13 \\
\hline 11 & $\mathrm{E}$ & 0.0885 & 2.587 & $0.826 \times 10^{2}$ & 0.335 & 0.68 & 0.09 & 0.40 & 1.33 & 0.11 \\
\hline 12 & $\mathrm{E}$ & 0.1083 & 3.071 & $0.494 \times 10^{2}$ & 0.338 & 0.55 & 0.03 & 0.32 & 1.49 & 0.03 \\
\hline 13 & $\mathrm{E}$ & 0.1335 & 3.538 & $0.306 \times 10^{2}$ & 0.330 & 0.82 & 0.02 & 0.25 & 0.91 & 0.04 \\
\hline 14 & $\mathrm{E}$ & 0.1664 & 4.051 & $0.191 \times 10^{2}$ & 0.321 & 0.54 & 0.04 & 0.19 & 1.32 & 0.03 \\
\hline 15 & $\mathrm{E}$ & 0.2107 & 4.702 & $0.108 \times 10^{2}$ & 0.298 & 0.57 & 0.05 & 0.15 & 1.02 & 0.05 \\
\hline 16 & $\mathrm{E}$ & 0.2726 & 5.685 & $0.520 \times 10$ & 0.262 & 0.66 & 0.04 & 0.12 & 2.03 & 0.00 \\
\hline 17 & $\mathrm{E}$ & 0.3679 & 9.154 & $0.101 \times 10$ & 0.189 & 0.69 & 0.05 & 0.25 & 1.40 & 0.02 \\
\hline 9 & $\mathrm{~F}$ & 0.0603 & 2.319 & $0.122 \times 10^{3}$ & 0.324 & 0.73 & 2.40 & 1.15 & 0.77 & 0.06 \\
\hline 10 & $\mathrm{~F}$ & 0.0734 & 2.806 & $0.705 \times 10^{2}$ & 0.329 & 0.65 & 2.17 & 0.87 & 0.48 & 0.03 \\
\hline 11 & $\mathrm{~F}$ & 0.0894 & 3.341 & $0.421 \times 10^{2}$ & 0.331 & 0.65 & 1.58 & 0.64 & 0.66 & 0.07 \\
\hline 12 & $\mathrm{~F}$ & 0.1094 & 3.946 & $0.255 \times 10^{2}$ & 0.331 & 0.58 & 0.96 & 0.51 & 1.03 & 0.07 \\
\hline 13 & $\mathrm{~F}$ & 0.1347 & 4.618 & $0.153 \times 10^{2}$ & 0.322 & 0.55 & 0.41 & 0.40 & 0.56 & 0.06 \\
\hline 14 & $\mathrm{~F}$ & 0.1679 & 5.344 & $0.930 \times 10$ & 0.311 & 0.56 & 0.11 & 0.30 & 1.45 & 0.02 \\
\hline 15 & $\mathrm{~F}$ & 0.2128 & 6.266 & $0.524 \times 10$ & 0.292 & 0.63 & 0.02 & 0.24 & 2.05 & 0.02 \\
\hline 16 & $\mathrm{~F}$ & 0.2759 & 7.501 & $0.251 \times 10$ & 0.249 & 0.57 & 0.04 & 0.22 & 0.85 & 0.03 \\
\hline
\end{tabular}

Table 6. - continued. 


\begin{tabular}{|c|c|c|c|c|c|c|c|}
\hline$x$ bin & $Q^{2}$ bin & $\langle x\rangle$ & $\left\langle Q^{2}\right\rangle$ & $\sigma^{d} / \sigma^{p}$ & $\delta_{\text {stat. }}$ & $\delta_{\text {rad. }}$ & $\delta_{\text {model }}$ \\
& & & {$\left[\mathrm{GeV}^{2}\right]$} & & {$[\%]$} & {$[\%]$} & {$[\%]$} \\
\hline 1 & $\mathrm{~A}$ & 0.0069 & 0.291 & 0.992 & 2.19 & 1.52 & 3.85 \\
2 & $\mathrm{~A}$ & 0.0102 & 0.404 & 0.952 & 1.93 & 0.94 & 2.53 \\
3 & $\mathrm{~A}$ & 0.0142 & 0.514 & 0.979 & 1.27 & 0.43 & 2.01 \\
4 & $\mathrm{~A}$ & 0.0186 & 0.626 & 0.984 & 1.41 & 0.39 & 1.71 \\
5 & $\mathrm{~A}$ & 0.0244 & 0.725 & 0.977 & 1.21 & 0.10 & 1.56 \\
6 & $\mathrm{~A}$ & 0.0325 & 0.872 & 0.965 & 1.26 & 0.57 & 1.47 \\
7 & $\mathrm{~A}$ & 0.0398 & 0.903 & 0.972 & 1.94 & 0.82 & 1.62 \\
8 & $\mathrm{~A}$ & 0.0486 & 0.929 & 0.962 & 3.24 & 0.54 & 2.70 \\
9 & $\mathrm{~A}$ & 0.0595 & 1.102 & 0.948 & 3.72 & 1.49 & 1.37 \\
10 & $\mathrm{~A}$ & 0.0724 & 1.127 & 0.936 & 3.69 & 0.63 & 1.17 \\
11 & $\mathrm{~A}$ & 0.0882 & 1.167 & 0.954 & 3.51 & 1.84 & 0.89 \\
12 & $\mathrm{~A}$ & 0.1078 & 1.205 & 0.923 & 5.02 & 1.51 & 0.81 \\
13 & $\mathrm{~A}$ & 0.1330 & 1.263 & 0.885 & 4.14 & 1.97 & 0.86 \\
14 & $\mathrm{~A}$ & 0.1657 & 1.337 & 0.927 & 3.57 & 2.48 & 1.09 \\
15 & $\mathrm{~A}$ & 0.2093 & 1.447 & 0.873 & 2.87 & 1.24 & 1.90 \\
16 & $\mathrm{~A}$ & 0.2642 & 1.749 & 0.848 & 1.72 & 1.09 & 2.76 \\
17 & $\mathrm{~A}$ & 0.3477 & 2.672 & 0.798 & 1.88 & 0.09 & 2.08 \\
18 & $\mathrm{~A}$ & 0.4605 & 4.224 & 0.783 & 1.59 & 0.19 & 1.77 \\
19 & $\mathrm{~A}$ & 0.6346 & 8.688 & 0.743 & 3.90 & 0.07 & 1.28 \\
1 & $\mathrm{~B}$ & 0.0085 & 0.354 & 0.968 & 1.97 & 0.69 & 2.97 \\
2 & $\mathrm{~B}$ & 0.0117 & 0.477 & 0.967 & 1.17 & 0.99 & 2.49 \\
3 & $\mathrm{~B}$ & 0.0152 & 0.612 & 0.972 & 0.93 & 0.81 & 2.28 \\
4 & $\mathrm{~B}$ & 0.0193 & 0.770 & 0.969 & 0.97 & 0.33 & 2.07 \\
5 & $\mathrm{~B}$ & 0.0250 & 0.896 & 0.981 & 0.78 & 0.27 & 1.69 \\
6 & $\mathrm{~B}$ & 0.0327 & 1.144 & 0.972 & 1.00 & 0.45 & 1.60 \\
7 & $\mathrm{~B}$ & 0.0401 & 1.074 & 0.964 & 1.56 & 0.32 & 1.38 \\
8 & $\mathrm{~B}$ & 0.0490 & 1.123 & 0.962 & 1.55 & 0.07 & 1.30 \\
9 & $\mathrm{~B}$ & 0.0596 & 1.281 & 0.969 & 2.96 & 0.57 & 0.87 \\
10 & $\mathrm{~B}$ & 0.0725 & 1.359 & 0.928 & 2.63 & 0.18 & 0.61 \\
11 & $\mathrm{~B}$ & 0.0884 & 1.399 & 0.925 & 2.71 & 0.14 & 0.55 \\
12 & $\mathrm{~B}$ & 0.1082 & 1.554 & 0.921 & 1.73 & 0.36 & 0.50 \\
13 & $\mathrm{~B}$ & 0.1333 & 1.632 & 0.901 & 2.57 & 0.73 & 0.53 \\
14 & $\mathrm{~B}$ & 0.1661 & 1.811 & 0.903 & 1.45 & 0.17 & 0.54 \\
15 & $\mathrm{~B}$ & 0.2104 & 2.050 & 0.885 & 2.13 & 0.25 & 0.61 \\
16 & $\mathrm{~B}$ & 0.2722 & 2.471 & 0.860 & 1.43 & 0.06 & 0.82 \\
17 & $\mathrm{~B}$ & 0.3620 & 3.790 & 0.809 & 1.21 & 0.09 & 0.60 \\
18 & $\mathrm{~B}$ & 0.4900 & 6.026 & 0.768 & 0.91 & 0.03 & 0.66 \\
19 & $\mathrm{~B}$ & 0.6641 & 12.778 & 0.756 & 3.22 & 0.09 & 0.67 \\
5 & $\mathrm{C}$ & 0.0270 & 1.092 & 0.990 & 1.04 & 0.43 & 2.03 \\
\hline
\end{tabular}

Table 7. Results on the inelastic Born cross-section ratio $\sigma^{d} / \sigma^{p}$. The statistical uncertainty $\delta_{\text {stat. }}$, the systematic uncertainty $\delta_{\text {rad. }}$ due to radiative corrections and $\delta_{\text {model }}$ due to the model dependence outside the acceptance are given in percent. Corresponding $x$ bin numbers and $Q^{2}$ bin numbers and the average values of $x$ and $Q^{2}$ are listed in the first four columns. The overall normalization uncertainty is $1.4 \%$. 


\begin{tabular}{|c|c|c|c|c|c|c|c|}
\hline$x$ bin & $Q^{2}$ bin & $\overline{\langle x\rangle}$ & $\begin{array}{c}\left\langle Q^{2}\right\rangle \\
{\left[\mathrm{GeV}^{2}\right]}\end{array}$ & $\sigma^{d} / \sigma^{p}$ & $\begin{array}{c}\delta_{\text {stat. }} \\
{[\%]}\end{array}$ & $\begin{array}{l}\delta_{\text {rad. }} \\
{[\%]}\end{array}$ & $\begin{array}{c}\delta_{\text {model }} \\
{[\%]}\end{array}$ \\
\hline 6 & $\mathrm{C}$ & 0.0338 & 1.391 & 0.990 & 1.49 & 0.73 & 2.15 \\
\hline 7 & C & 0.0401 & 1.293 & 0.975 & 1.18 & 0.07 & 1.45 \\
\hline 8 & $\mathrm{C}$ & 0.0490 & 1.460 & 0.952 & 1.02 & 0.19 & 1.12 \\
\hline 9 & C & 0.0596 & 1.466 & 0.941 & 1.59 & 0.20 & 0.75 \\
\hline 10 & $\mathrm{C}$ & 0.0726 & 1.627 & 0.943 & 1.59 & 0.26 & 0.53 \\
\hline 11 & $\mathrm{C}$ & 0.0885 & 1.691 & 0.945 & 1.60 & 0.08 & 0.46 \\
\hline 12 & C & 0.1082 & 2.013 & 0.917 & 1.39 & 0.05 & 0.39 \\
\hline 13 & $\mathrm{C}$ & 0.1334 & 2.158 & 0.920 & 1.57 & 0.17 & 0.36 \\
\hline 14 & C & 0.1662 & 2.458 & 0.891 & 1.74 & 0.10 & 0.33 \\
\hline 15 & $\mathrm{C}$ & 0.2105 & 2.851 & 0.862 & 1.07 & 0.01 & 0.31 \\
\hline 16 & $\mathrm{C}$ & 0.2724 & 3.479 & 0.851 & 0.96 & 0.08 & 0.30 \\
\hline 17 & $\mathrm{C}$ & 0.3622 & 5.125 & 0.813 & 0.87 & 0.03 & 0.22 \\
\hline 18 & $\mathrm{C}$ & 0.4957 & 8.280 & 0.760 & 0.97 & 0.02 & 0.23 \\
\hline 7 & $\mathrm{D}$ & 0.0408 & 1.615 & 0.969 & 1.01 & 0.51 & 1.94 \\
\hline 8 & D & 0.0496 & 1.927 & 0.978 & 0.90 & 0.24 & 1.63 \\
\hline 9 & D & 0.0596 & 1.675 & 0.961 & 1.76 & 0.22 & 0.74 \\
\hline 10 & D & 0.0726 & 1.928 & 0.951 & 2.11 & 0.12 & 0.51 \\
\hline 11 & D & 0.0885 & 2.080 & 0.946 & 1.31 & 0.10 & 0.43 \\
\hline 12 & D & 0.1083 & 2.490 & 0.914 & 1.46 & 0.31 & 0.34 \\
\hline 13 & $\mathrm{D}$ & 0.1334 & 2.795 & 0.923 & 1.20 & 0.21 & 0.29 \\
\hline 14 & $\mathrm{D}$ & 0.1663 & 3.195 & 0.896 & 1.29 & 0.10 & 0.25 \\
\hline 15 & $\mathrm{D}$ & 0.2106 & 3.709 & 0.877 & 0.99 & 0.10 & 0.21 \\
\hline 16 & D & 0.2726 & 4.527 & 0.834 & 1.25 & 0.02 & 0.18 \\
\hline 17 & D & 0.3624 & 6.797 & 0.808 & 0.82 & 0.02 & 0.13 \\
\hline 18 & D & 0.5038 & 11.344 & 0.770 & 1.43 & 0.02 & 0.31 \\
\hline 9 & $\mathrm{E}$ & 0.0597 & 1.906 & 0.950 & 1.31 & 0.18 & 0.81 \\
\hline 10 & $\mathrm{E}$ & 0.0726 & 2.276 & 0.954 & 1.34 & 0.20 & 0.58 \\
\hline 11 & $\mathrm{E}$ & 0.0885 & 2.587 & 0.944 & 1.00 & 0.14 & 0.44 \\
\hline 12 & $\mathrm{E}$ & 0.1083 & 3.071 & 0.940 & 0.81 & 0.04 & 0.35 \\
\hline 13 & $\mathrm{E}$ & 0.1335 & 3.538 & 0.912 & 1.20 & 0.04 & 0.27 \\
\hline 14 & $\mathrm{E}$ & 0.1664 & 4.051 & 0.901 & 0.79 & 0.06 & 0.21 \\
\hline 15 & $\mathrm{E}$ & 0.2107 & 4.702 & 0.878 & 0.82 & 0.04 & 0.17 \\
\hline 16 & $\mathrm{E}$ & 0.2726 & 5.685 & 0.853 & 0.95 & 0.04 & 0.13 \\
\hline 17 & $\mathrm{E}$ & 0.3679 & 9.154 & 0.802 & 0.98 & 0.02 & 0.26 \\
\hline 9 & $\mathrm{~F}$ & 0.0603 & 2.319 & 0.969 & 1.07 & 0.14 & 1.26 \\
\hline 10 & $\mathrm{~F}$ & 0.0734 & 2.806 & 0.955 & 0.96 & 0.15 & 0.94 \\
\hline 11 & $\mathrm{~F}$ & 0.0894 & 3.341 & 0.936 & 0.96 & 0.09 & 0.72 \\
\hline 12 & $\mathrm{~F}$ & 0.1094 & 3.946 & 0.947 & 0.85 & 0.08 & 0.56 \\
\hline 13 & $\mathrm{~F}$ & 0.1347 & 4.618 & 0.920 & 0.81 & 0.05 & 0.44 \\
\hline 14 & $\mathrm{~F}$ & 0.1679 & 5.344 & 0.898 & 0.81 & 0.03 & 0.32 \\
\hline 15 & $\mathrm{~F}$ & 0.2128 & 6.266 & 0.874 & 0.91 & 0.01 & 0.26 \\
\hline 16 & $\mathrm{~F}$ & 0.2759 & 7.501 & 0.829 & 0.82 & 0.04 & 0.23 \\
\hline
\end{tabular}

Table 7. - continued. 


\begin{tabular}{|c|c|cc|cc|cc|}
\hline Parameter & ALLM97-P & \multicolumn{2}{|c|}{ GD07-P } & \multicolumn{2}{c|}{ GD11-P } & \multicolumn{2}{c|}{ GD11-D } \\
& value & value & unc. & value & unc. & value & unc. \\
\hline$m_{0}^{2}\left[\mathrm{GeV}^{2}\right]$ & 0.31985 & 0.454 & 0.0283 & 0.5063 & 0.0236 & 0.426 & - \\
$m_{\mathcal{P}}^{2}\left[\mathrm{GeV}^{2}\right]$ & 49.457 & 30.7 & 2.85 & 34.75 & 2.56 & 0.00007713 & 0.000319 \\
$m_{\mathcal{R}}^{2}\left[\mathrm{GeV}^{2}\right]$ & 0.15052 & 0.117 & 0.0465 & 0.03190 & 0.00828 & 0.2293 & 0.0763 \\
$Q_{0}^{2}\left[\mathrm{GeV}^{2}\right]$ & 0.52544 & 1.15 & 0.358 & 1.374 & 0.349 & 2.65 & - \\
$\Lambda_{0}^{2}\left[\mathrm{GeV}^{2}\right]$ & 0.06527 & 0.06527 & - & 0.06527 & - & 0.06527 & - \\
$a_{\mathcal{P} 1}$ & -0.0808 & -0.105 & 0.00507 & -0.11895 & 0.00411 & -0.4287 & 0.0773 \\
$a_{\mathcal{P} 2}$ & 0.44812 & -0.495 & 0.0306 & -0.4783 & 0.0224 & 0.2891 & 0.0646 \\
$a_{\mathcal{P} 3}$ & 1.1709 & 1.29 & 0.243 & 1.353 & 0.203 & 0.3931 & 0.0935 \\
$b_{\mathcal{P} 4}$ & 0.36292 & -1.42 & 0.491 & 1.0833 & 0.0908 & -27.212 & 0.649 \\
$b_{\mathcal{P} 5}$ & 1.8917 & 4.51 & 0.540 & 2.656 & 0.363 & 30.687 & 0.650 \\
$b_{\mathcal{P} 6}$ & 1.8439 & 0.551 & 0.120 & 1.771 & 0.204 & 0.04577 & 0.00198 \\
$c_{\mathcal{P} 7}$ & 0.28067 & 0.339 & 0.0194 & 0.3638 & 0.0140 & 0.00073 & 0.00181 \\
$c_{\mathcal{P} 8}$ & 0.22291 & 0.127 & 0.0217 & 0.1211 & 0.0163 & 0.9741 & 0.0566 \\
$c_{\mathcal{P} 9}$ & 2.1979 & 1.16 & 0.246 & 1.166 & 0.197 & 0.8722 & 0.0800 \\
$a_{\mathcal{R} 1}$ & 0.584 & 0.374 & 0.0320 & 0.3425 & 0.0243 & 0.2986 & 0.0237 \\
$a_{\mathcal{R} 2}$ & 0.37888 & 0.998 & 0.102 & 1.0603 & 0.0640 & 3.615 & 0.230 \\
$a_{\mathcal{R} 3}$ & 2.6063 & 0.775 & 0.112 & 0.5164 & 0.0440 & 1.1455 & 0.0653 \\
$b_{\mathcal{R} 4}$ & 0.01147 & 2.71 & 0.393 & -10.408 & 0.627 & 1.987 & 0.333 \\
$b_{\mathcal{R} 5}$ & 3.7582 & 1.83 & 0.537 & 14.857 & 0.627 & 7.150 & 0.343 \\
$b_{\mathcal{R} 6}$ & 0.49338 & 1.26 & 0.296 & 0.07739 & 0.00861 & 0.9350 & 0.0935 \\
$c_{\mathcal{R} 7}$ & 0.80107 & 0.838 & 0.106 & 1.3633 & 0.0867 & 1.0316 & 0.0783 \\
$c_{\mathcal{R} 8}$ & 0.97307 & 2.36 & 0.557 & 2.256 & 0.503 & 26.36 & 6.19 \\
$c_{\mathcal{R} 9}$ & 3.4942 & 1.77 & 0.209 & 2.209 & 0.260 & 3.024 & 0.136 \\
\hline
\end{tabular}

Table 8. Results of fits to the cross section $\sigma_{L+T}$ using the ALLM functional form [3]. Parameters of the ALLM97 fit [25] and the GD07-P fit [51] (both based on proton data) are quoted together with the new parameters determined from current world data including the HERMES results discussed in this paper on proton (GD11-P) and deuteron (GD11-D) cross sections. In ref. [51] the uncertainties were calculated with an UP value [49] equal to 24.7. That identical fit was redone here with the same value of $\mathrm{UP}=1$ as the new fits in order to provide an appropriate comparison of its uncertainties. The ALLM97 fit provided no uncertainties. The uncertainties given in the table for GD07-P, GD11$\mathrm{P}$, and GD11-D correspond to the diagonal elements of the full covariance matrix which must be used to calculate uncertainties in $F_{2}$ or cross sections. They can be obtained by contacting the management of the HERMES collaboration: < management@hermes.desy.de>. The parameter $\Lambda_{0}^{2}$ has no uncertainty as it is fixed in the fits. In the case of the deuteron fit, the parameters $m_{0}^{2}$ and $Q_{0}^{2}$ had to be fixed in order to obtain a good fit. 


\begin{tabular}{|l|c|c|}
\hline Data set & GD11-P & GD11-D \\
\hline HERA (positron beam) $[44]$ & -0.0065 & - \\
HERA (electron beam) [44] & -0.0067 & - \\
E665 [18] & 0.020 & -0.014 \\
NMC-90 GeV [17] & -0.00020 & -0.029 \\
NMC-120 GeV [17] & 0.011 & -0.0096 \\
NMC-200 GeV [17] & 0.0093 & 0.0036 \\
NMC-280 GeV [17] & 0.0035 & 0.0023 \\
BCDMS-100 GeV [15] & -0.032 & - \\
BCDMS-120 GeV [15] & -0.028 & -0.0075 \\
BCDMS-200 GeV [15] & -0.027 & -0.0060 \\
BCDMS-280 GeV [15] & -0.023 & -0.0031 \\
SLAC E49a [19] & 0.016 & -0.0013 \\
SLAC E49b [19] & 0.022 & 0.0062 \\
SLAC E61 [19] & 0.016 & 0.0070 \\
SLAC E87 [19] & 0.016 & 0.0045 \\
SLAC E89a [19] & 0.036 & 0.0087 \\
SLAC E89b [19] & 0.018 & 0.00081 \\
SLAC E139 [19] & - & 0.0014 \\
SLAC E140 [19] & - & 0.0025 \\
JLAB [22] & -0.012 & -0.040 \\
JLAB [20] & -0.0063 & - \\
JLAB [21] & - & -0.0012 \\
JLAB (Rosenbluth) [23] & 0.0014 & 0.0088 \\
JLAB (Model Dependent) 23$]$ & 0.0085 & 0.0088 \\
HERMES (T.A.) & 0.015 & -0.022 \\
\hline
\end{tabular}

Table 9. Normalizations $\delta_{k} \nu_{k}$ (see eq. (6.2)) obtained in the GD11 fits.

\section{Author list}

A. Airapetian, ${ }^{12,15}$ N. Akopov, ${ }^{26}$ Z. Akopov, ${ }^{5}$ E.C. Aschenauer, ${ }^{6, a}$ W. Augustyniak, ${ }^{25}$ R. Avakian, ${ }^{26}$ A. Avetissian, ${ }^{26}$ E. Avetisyan, ${ }^{5}$ S. Belostotski, ${ }^{18}$ N. Bianchi, ${ }^{10}$ H.P. Blok, ${ }^{17,24}$ A. Borissov, ${ }^{5}$ J. Bowles, ${ }^{13}$ V. Bryzgalov, ${ }^{19}$ J. Burns, ${ }^{13}$ M. Capiluppi, ${ }^{9}$ G.P. Capitani, ${ }^{10}$ E. Cisbani, ${ }^{21}$ G. Ciullo, ${ }^{9}$ M. Contalbrigo, ${ }^{9}$ P.F. Dalpiaz, ${ }^{9}$ W. Deconinck, ${ }^{5}$ R. De Leo,${ }^{2}$ L. De Nardo, ${ }^{11,5}$ E. De Sanctis, ${ }^{10}$ M. Diefenthaler, ${ }^{14,8}$ P. Di Nezza, ${ }^{10}$

\footnotetext{
${ }^{a}$ Now at: Brookhaven National Laboratory, Upton, New York 11772-5000, U.S.A.
} 
M. Düren, ${ }^{12}$ M. Ehrenfried, ${ }^{12}$ G. Elbakian, ${ }^{26}$ F. Ellinghaus, ${ }^{4}$ R. Fabbri, ${ }^{6}$ A. Fantoni, ${ }^{10}$ L. Felawka, ${ }^{22}$ S. Frullani, ${ }^{21}$ D. Gabbert, ${ }^{6}$ G. Gapienko, ${ }^{19}$ V. Gapienko, ${ }^{19}$ F. Garibaldi, ${ }^{21}$ G. Gavrilov, ${ }^{5,18,22}$ V. Gharibyan, ${ }^{26}$ F. Giordano, ${ }^{5,9}$ S. Gliske, ${ }^{15}$ M. Golembiovskaya, ${ }^{6}$ C. Hadjidakis, ${ }^{10}$ M. Hartig, ${ }^{5, b}$ D. Hasch, ${ }^{10}$ G. Hill, ${ }^{13}$ A. Hillenbrand, ${ }^{6}$ M. Hoek, ${ }^{13}$ Y. Holler,${ }^{5}$ I. Hristova, ${ }^{6}$ Y. Imazu, ${ }^{23}$ A. Ivanilov, ${ }^{19}$ H.E. Jackson, ${ }^{1}$ H.S. Jo, ${ }^{11}$ S. Joosten, ${ }^{14,11}$ R. Kaiser, ${ }^{13, c}$ G. Karyan, ${ }^{26}$ T. Keri, ${ }^{13,12}$ E. Kinney, ${ }^{4}$ A. Kisselev, ${ }^{18}$ V. Korotkov, ${ }^{19}$ V. Kozlov, ${ }^{16}$ P. ${\text { Kravchenko, }{ }^{8,18} \text { V.G. }}$ Krivokhijine, ${ }^{7}$ L. Lagamba, ${ }^{2}$ R. Lamb, ${ }^{14}$ L. Lapikás, ${ }^{17}$ I. Lehmann, ${ }^{13}$ P. Lenisa, ${ }^{9}$ L.A. LindenLevy, ${ }^{14}$ A. López Ruiz, ${ }^{11}$ W. Lorenzon, ${ }^{15}$ X.-G. Lu, ${ }^{6}$ X.-R. Lu, ${ }^{23}$ B.-Q. Ma,${ }^{3}$ D. Mahon, ${ }^{13}$ N.C.R. Makins, ${ }^{14}$ S.I. Manaenkov, ${ }^{18}$ L. Manfré, ${ }^{21}$ Y. Mao, ${ }^{3}$ B. Marianski, ${ }^{25}$ A. Martinez de la Ossa, ${ }^{8,4}$ H. Marukyan, ${ }^{26}$ C.A. Miller, ${ }^{22}$ Y. Miyachi, ${ }^{23}$ A. Movsisyan, ${ }^{26}$ V. Muccifora ${ }^{10}$ M. Murray, ${ }^{13}$ A. Mussgiller,${ }^{5,8}$ E. Nappi, ${ }^{2}$ Y. Naryshkin, ${ }^{18}$ A. Nass, ${ }^{8}$ M. Negodaev, ${ }^{6}$ W.-D. Nowak, ${ }^{6}$ L.L. Pappalardo, ${ }^{9}$ R. Perez-Benito, ${ }^{12}$ N. Pickert,${ }^{8}$ M. Raithel,${ }^{8}$ P.E. Reimer,${ }^{1}$ A.R. Reolon, ${ }^{10}$ C. Riedl, ${ }^{6}$ K. Rith,${ }^{8}$ G. Rosner, ${ }^{13}$ A. Rostomyan, ${ }^{5}$ J. Rubin, ${ }^{14}$ D. Ryckbosch, ${ }^{11}$ Y. Salomatin, ${ }^{19}$ F. Sanftl, ${ }^{23,20}$ A. Schäfer, ${ }^{20}$ G. Schnell, $, 11, \mathrm{~d}$ K.P. Schüler, ${ }^{5}$ B. Seitz, ${ }^{13}$ T.-A. Shibata, ${ }^{23}$ V. Shutov, ${ }^{7}$ M. Stancari, ${ }^{9}$ M. Statera, ${ }^{9}$ E. Steffens, ${ }^{8}$ J.J.M. Steijger, ${ }^{17}$ H. Stenzel, ${ }^{12}$ J. Stewart, ${ }^{6}$ F. Stinzing, ${ }^{8}$ S. Taroian, ${ }^{26}$ A. Trzcinski,${ }^{25}$ M. Tytgat,${ }^{11}$ A. Vandenbroucke,${ }^{11}$ Y. Van Haarlem, ${ }^{11}$ C. Van Hulse, ${ }^{11}$ D. Veretennikov, ${ }^{18}$ V. Vikhrov, ${ }^{18}$ I. Vilardi, ${ }^{2}$ C. Vogel, ${ }^{8}$ S. Wang, ${ }^{3}$ S. Yaschenko, ${ }^{6,8}$ H. Ye, ${ }^{3}$ Z. Ye, ${ }^{5}$ S. Yen, ${ }^{22}$ W. Yu, ${ }^{12}$ D. Zeiler, ${ }^{8}$ B. Zihlmann ${ }^{5}$ and P. Zupranski ${ }^{25}$

${ }^{1}$ Physics Division, Argonne National Laboratory, Argonne, Illinois 60439-4843, U.S.A.

${ }^{2}$ Istituto Nazionale di Fisica Nucleare, Sezione di Bari, 70124 Bari, Italy

${ }^{3}$ School of Physics, Peking University, Beijing 100871, China

${ }^{4}$ Nuclear Physics Laboratory, University of Colorado, Boulder, Colorado 80309-0390, U.S.A.

${ }^{5}$ DESY, 22603 Hamburg, Germany

${ }^{6}$ DESY, 15738 Zeuthen, Germany

${ }^{7}$ Joint Institute for Nuclear Research, 141980 Dubna, Russia

${ }^{8}$ Physikalisches Institut, Universität Erlangen-Nürnberg, 91058 Erlangen, Germany

${ }^{9}$ Istituto Nazionale di Fisica Nucleare, Sezione di Ferrara and Dipartimento di Fisica,

Università di Ferrara, 44100 Ferrara, Italy

${ }^{10}$ Istituto Nazionale di Fisica Nucleare, Laboratori Nazionali di Frascati, 00044 Frascati, Italy

${ }^{11}$ Department of Subatomic and Radiation Physics, University of Gent, 9000 Gent, Belgium

${ }^{12}$ Physikalisches Institut, Universität Gießen, 35392 Gießen, Germany

${ }^{13}$ Department of Physics and Astronomy, University of Glasgow, Glasgow G12 8QQ, U.K.

${ }^{14}$ Department of Physics, University of Illinois, Urbana, Illinois 61801-3080, U.S.A.

\footnotetext{
${ }^{\text {b} N o w ~ a t: ~ I n s t i t u t ~ f u ̈ r ~ K e r n p h y s i k, ~ U n i v e r s i t a ̈ t ~ F r a n k f u r t ~ a . M ., ~} 60438$ Frankfurt a.M., Germany.

${ }^{\mathrm{c}}$ Present address: International Atomic Energy Agency, A-1400 Vienna, Austria.

${ }^{\mathrm{d} N o w}$ at: Department of Theoretical Physics, University of the Basque Country UPV/EHU, 48080 Bilbao, Spain and IKERBASQUE, Basque Foundation for Science, 48011 Bilbao, Spain.
} 
${ }^{15}$ Randall Laboratory of Physics, University of Michigan, Ann Arbor, Michigan 48109-1040, U.S.A.

${ }^{16}$ Lebedev Physical Institute, 117924 Moscow, Russia

${ }^{17}$ National Institute for Subatomic Physics (Nikhef), 1009 DB Amsterdam, The Netherlands

${ }^{18}$ Petersburg Nuclear Physics Institute, Gatchina, Leningrad region 188300, Russia

${ }^{19}$ Institute for High Energy Physics, Protvino, Moscow region 142281, Russia

${ }^{20}$ Institut für Theoretische Physik, Universität Regensburg, 93040 Regensburg, Germany

${ }^{21}$ Istituto Nazionale di Fisica Nucleare, Sezione di Roma, Gruppo Collegato Sanità and Istituto Superiore di Sanità, 00161 Roma, Italy

${ }^{22}$ TRIUMF, Vancouver, British Columbia V6T 2A3, Canada

${ }^{23}$ Department of Physics, Tokyo Institute of Technology, Tokyo 152, Japan

${ }^{24}$ Department of Physics, VU University, $1081 \mathrm{HV}$ Amsterdam, The Netherlands

${ }^{25}$ Andrzej Soltan Institute for Nuclear Studies, 00-689 Warsaw, Poland

${ }^{26}$ Yerevan Physics Institute, 375036 Yerevan, Armenia

Open Access. This article is distributed under the terms of the Creative Commons Attribution Noncommercial License which permits any noncommercial use, distribution, and reproduction in any medium, provided the original author(s) and source are credited.

\section{References}

[1] R. Devenish and A. Cooper-Sarkar, Deep inelastic scattering, Oxford University Press, Oxford U.K. (2004).

[2] B. Badelek and J. Kwiecinski, Low $Q^{2}$, low $x$ region in electroproduction, Rev. Mod. Phys. 68 (1996) 445 [hep-ph/9408318] [SPIRES].

[3] H. Abramowicz, E.M. Levin, A. Levy and U. Maor, A parametrization of $\sigma_{T}\left(\gamma^{*} p\right)$ above the resonance region $Q^{2} \geq 0$, Phys. Lett. B 269 (1991) 465 [SPIRES].

[4] H1 collaboration, S. Aid et al., A measurement and QCD analysis of the proton structure function $F_{2}\left(x, Q^{2}\right)$ at HERA, Nucl. Phys. B 470 (1996) 3 [hep-ex/9603004] [SPIRES].

[5] H1 collaboration, C. Adloff et al., A measurement of the proton structure function $F_{2}\left(x, Q^{2}\right)$ at low $x$ and low $Q^{2}$ at HERA, Nucl. Phys. B 497 (1997) 3 [hep-ex/9703012] [SPIRES].

[6] H1 collaboration, C. Adloff et al., Measurement of neutral and charged current cross-sections in positron proton collisions at large momentum transfer, Eur. Phys. J. C 13 (2000) 609 [hep-ex/9908059] [SPIRES].

[7] H1 collaboration, C. Adloff et al., Measurement of neutral and charged current cross-sections in electron-proton collisions at high $Q^{2}$, Eur. Phys. J. C 19 (2001) 269 [hep-ex/0012052] [SPIRES].

[8] H1 collaboration, C. Adloff et al., Measurement and QCD analysis of neutral and charged current cross sections at HERA, Eur. Phys. J. C 30 (2003) 1 [hep-ex/0304003] [SPIRES].

[9] ZEUS collaboration, M. Derrick et al., Measurement of the proton structure function $F_{2}$ in $e$ p scattering at HERA, Phys. Lett. B 316 (1993) 412 [SPIRES]. 
[10] ZEUS collaboration, M. Derrick et al., Measurement of the proton structure function $F_{2}$ at low $x$ and low $Q^{2}$ at HERA, Z. Phys. C 69 (1996) 607 [hep-ex/9510009] [SPIRES].

[11] ZEUS collaboration, M. Derrick et al., Measurement of the $F_{2}$ structure function in deep inelastic $e^{+} p$ scattering using 1994 data from the ZEUS detector at HERA,

Z. Phys. C 72 (1996) 399 [hep-ex/9607002] [SPIRES].

[12] ZEUS collaboration, J. Breitweg et al., Measurement of the proton structure function $F_{2}$ and $\sigma_{\text {tot }}\left(\gamma^{*} p\right)$ at low $Q^{2}$ and very low $x$ at HERA, Phys. Lett. B 407 (1997) 432 [hep-ex/9707025] [SPIRES].

[13] ZEUS collaboration, J. Breitweg et al., Measurement of the proton structure function $F_{2}$ at very low $Q^{2}$ at HERA, Phys. Lett. B 487 (2000) 53 [hep-ex/0005018] [SPIRES].

[14] ZEUS collaboration, S. Chekanov et al., Measurement of the neutral current cross section and $F_{2}$ structure function for deep inelastic $e^{+} p$ scattering at HERA,

Eur. Phys. J. C 21 (2001) 443 [hep-ex/0105090] [SPIRES].

[15] BCDMS collaboration, A.C. Benvenuti et al., A high statistics measurement of the proton structure functions $F_{2}\left(x, Q^{2}\right)$ and $R$ from deep inelastic muon scattering at high $Q^{2}$, Phys. Lett. B 223 (1989) 485 [SPIRES].

[16] European Muon collaboration, J.J. Aubert et al., Measurements of the nucleon structure functions $\mathrm{F}_{2} \mathrm{~N}$ in deep inelastic muon scattering from deuterium and comparison with those from hydrogen and iron, Nucl. Phys. B 293 (1987) 740 [SPIRES].

[17] New Muon collaboration, M. Arneodo et al., Measurement of the proton and deuteron structure functions, $F_{2}^{p}$ and $F_{2}^{d}$ and of the ratio $\sigma_{L} / \sigma_{T}$, Nucl. Phys. B 483 (1997) 3 [hep-ph/9610231] [SPIRES].

[18] E665 collaboration, M.R. Adams et al., Proton and deuteron structure functions in muon scattering at $470 \mathrm{GeV}$, Phys. Rev. D 54 (1996) 3006 [SPIRES].

[19] L.W. Whitlow, Deep inelastic structure functions from electron scattering on hydrogen, deuterium, and iron at $0.6 \mathrm{GeV}^{2} \leq Q^{2} \leq 30 \mathrm{GeV}^{2}$, Ph.D. thesis, SLAC-0357, California University, Stanford U.S.A. (1990) [SPIRES].

[20] CLAS collaboration, M. Osipenko et al., The proton structure function $F_{2}$ with CLAS, hep-ex/0309052 [SPIRES].

[21] CLAS collaboration, M. Osipenko et al., Measurement of the deuteron structure function $F_{2}$ in the resonance region and evaluation of its moments, Phys. Rev. C 73 (2006) 045205 [hep-ex/0506004] [SPIRES].

[22] Jefferson Lab E00-115 collaboration, S.P. Malace et al., Applications of quark-hadron duality in $F_{2}$ structure function, Phys. Rev. C 80 (2009) 035207 [arXiv:0905.2374] [SPIRES].

[23] V. Tvaskis et al., The proton and deuteron $F_{2}$ structure function at low $Q^{2}$, Phys. Rev. C 81 (2010) 055207 [arXiv:1002.1669] [SPIRES].

[24] HERMES collaboration, K. Ackerstaff et al., The HERMES spectrometer, Nucl. Instrum. Meth. A 417 (1998) 230 [hep-ex/9806008] [SPIRES].

[25] H. Abramowicz and A. Levy, The ALLM parameterization of $\sigma_{\mathrm{tot}}\left(\gamma^{*} p\right)$ : an update, hep-ph/9712415 [SPIRES]. 
[26] F. Close, An introduction to quarks and partons, Academic Press, London U.K. (1979) [SPIRES].

[27] L.N. Hand, Experimental investigation of pion electroproduction, Phys. Rev. 129 (1963) 1834 [SPIRES].

[28] H. Abramowicz and A. Caldwell, HERA collider physics, Rev. Mod. Phys. 71 (1999) 1275 [hep-ex/9903037] [SPIRES].

[29] N. Akopov et al., The HERMES dual-radiator ring imaging Cerenkov detector, Nucl. Instrum. Meth. A 479 (2002) 511 [physics/0104033] [SPIRES].

[30] E. Aschenauer et al., Optical characterization of $n=1.03$ silica aerogel used as radiator in the RICH of HERMES, Nucl. Instrum. Meth. A 440 (2000) 338 [SPIRES].

[31] T. Benisch et al., The luminosity monitor of the HERMES experiment at DESY, Nucl. Instrum. Meth. A 471 (2001) 314 [SPIRES].

[32] E143 collaboration, K. Abe et al., Measurements of $R=\sigma_{L} / \sigma_{T}$ for $0.03<x<0.1$ and fit to world data, Phys. Lett. B 452 (1999) 194 [hep-ex/9808028] [SPIRES].

[33] L.W. Whitlow, S. Rock, A. Bodek, E.M. Riordan and S. Dasu, A precise extraction of $R=\sigma_{L} / \sigma_{T}$ from a global analysis of the SLAC deep inelastic e-p and e-d scattering cross-sections, Phys. Lett. B 250 (1990) 193 [SPIRES].

[34] New Muon collaboration, P. Amaudruz et al., The ratio $F_{2}^{n} / F_{2}^{p}$ in deep inelastic muon scattering, Nucl. Phys. B 371 (1992) 3 [SPIRES].

[35] I. Akushevich, H. Böttcher and D. Ryckbosch, RADGEN 1.0: Monte Carlo generator for radiative events in DIS on polarized and unpolarized targets, hep-ph/9906408 [SPIRES].

[36] S.I. Bilenkaya, S.M. Bilenkii, Y.M. Kazarinov and L.I. Lapidus, The proton electromagnetic form factor and heavy hypothetical particles, Pisma Zh. Eksp. Teor. Fiz. 19 (1973) 613 [SPIRES].

[37] G. Höhler et al., Analysis of electromagnetic nucleon form-factors, Nucl. Phys. B 114 (1976) 505 [SPIRES].

[38] J. Arrington, W. Melnitchouk and J.A. Tjon, Global analysis of proton elastic form factor data with two-photon exchange corrections, Phys. Rev. C 76 (2007) 035205 [arXiv:0707.1861] [SPIRES].

[39] HERMES collaboration, A. Airapetian et al., Precise determination of the spin structure function $g_{1}$ of the proton, deuteron and neutron, Phys. Rev. D 75 (2007) 012007 [hep-ex/0609039] [SPIRES].

[40] HERMES collaboration, K. Ackerstaff et al., Nuclear effects on $R=\sigma_{L} / \sigma_{T}$ in deep inelastic scattering, Phys. Lett. B 475 (2000) 386 [Erratum ibid. B 567 (2003) 339] [hep-ex/9910071] [SPIRES].

[41] HERMES collaboration, A. Airapetian et al., Measurement of the proton spin structure function $g_{1}^{p}$ with a pure hydrogen target, Phys. Lett. B 442 (1998) 484 [hep-ex/9807015] [SPIRES].

[42] New Muon collaboration, P. Amaudruz et al., Gottfried sum from the ratio $F_{2}^{n} / F_{2}^{p}$, Phys. Rev. Lett. 66 (1991) 2712 [SPIRES]. 
[43] New Muon collaboration, M. Arneodo et al., Reevaluation of the Gottfried sum, Phys. Rev. D 50 (1994) 1 [SPIRES].

[44] H1 AND ZEUS collaboration, F.D. Aaron et al., Combined measurement and QCD analysis of the inclusive $e^{ \pm} p$ scattering cross sections at HERA, JHEP 01 (2010) 109 [arXiv:0911.0884] [SPIRES].

[45] Particle Data Group collaboration, K. Nakamura et al., Review of particle physics, J. Phys. G 37 (2010) 075021 [SPIRES].

[46] A.D. Martin, R.G. Roberts, W.J. Stirling and R.S. Thorne, MRST2001: partons and $\alpha_{s}$ from precise deep inelastic scattering and Tevatron jet data, Eur. Phys. J. C 23 (2002) 73 [hep-ph/0110215] [SPIRES].

[47] R. Wallny, A measurement of the gluon distribution in the proton and of the strong coupling constant $\alpha_{s}$ from inclusive deep-inelastic scattering, Ph.D. thesis, Universität Zürich, Zürich Switzerland (2001) [SPIRES].

[48] D. Stump et al., Uncertainties of predictions from parton distribution functions. I. The Lagrange multiplier method, Phys. Rev. D 65 (2001) 014012 [hep-ph/0101051] [SPIRES].

[49] F. James, MINUIT, reference manual, version 94.1, CERN program library long writeup D506 (European Organization for Nuclear Research, Geneva, 1994), MINUIT, CERN Program Library Long Writeup D506, CERN, Geneva Switzerland (1994).

[50] J. Pumplin, D.R. Stump and W.K. Tung, Multivariate fitting and the error matrix in global analysis of data, Phys. Rev. D 65 (2001) 014011 [hep-ph/0008191] [SPIRES].

[51] D. Gabbert and L. De Nardo, New global fit to the total photon-proton cross-section $\sigma_{L+T}$ and to the structure function $F_{2}$, arXiv:0708.3196 [SPIRES].

[52] New Muon collaboration, M. Arneodo et al., Accurate measurement of $F_{2}^{d} / F_{2}^{p}$ and $R^{d}-R^{p}$, Nucl. Phys. B 487 (1997) 3 [hep-ex/9611022] [SPIRES]. 